\title{
Construction of Time-Stamped Mobility Map for Path Tracking via Smith-Waterman Measurement Matching
}

\author{
Mu Zhou, ${ }^{1,2}$ Zengshan Tian, ${ }^{1}$ Kunjie Xu, ${ }^{3}$ Haibo Wu, ${ }^{4}$ Qiaolin Pu, ${ }^{1}$ and Xiang Yu ${ }^{1}$ \\ ${ }^{1}$ Chongqing Key Lab of Mobile Communications Technology, Chongqing University of Posts and Telecommunications, \\ Chongqing 400065, China \\ ${ }^{2}$ Department of Electronic and Computer Engineering, The Hong Kong University of Science and Technology, Hong Kong \\ ${ }^{3}$ Graduate Telecommunications and Networking Program, The University of Pittsburgh, Pittsburgh, PA 15260, USA \\ ${ }^{4}$ China Internet Research Lab, China Science and Technology Network, Computer Network Information Center, \\ Chinese Academy of Sciences, Beijing 100190, China \\ Correspondence should be addressed to Mu Zhou; zhoumu@cqupt.edu.cn
}

Received 26 October 2013; Revised 18 January 2014; Accepted 19 January 2014; Published 17 March 2014

Academic Editor: Cristian Toma

Copyright (c) $2014 \mathrm{Mu}$ Zhou et al. This is an open access article distributed under the Creative Commons Attribution License, which permits unrestricted use, distribution, and reproduction in any medium, provided the original work is properly cited.

\begin{abstract}
Path tracking in wireless and mobile environments is a fundamental technology for ubiquitous location-based services (LBSs). In particular, it is very challenging to develop highly accurate and cost-efficient tracking systems applied to the anonymous areas where the floor plans are not available for security and privacy reasons. This paper proposes a novel path tracking approach for large WiFi areas based on the time-stamped unlabeled mobility map which is constructed from Smith-Waterman received signal strength (RSS) measurement matching. Instead of conventional location fingerprinting, we construct mobility map with the technique of dimension reduction from the raw measurement space into a low-dimensional embedded manifold. The feasibility of our proposed approach is verified by the real-world experiments in the HKUST campus Wi-Fi networks, sMobileNet. The experimental results prove that our approach is adaptive and capable of achieving an adequate precision level in path tracking.
\end{abstract}

\section{Introduction}

The recent decade has witnessed a growing interest in the location-based applications and services for both indoor and outdoor environments [1-4]. Since the Wi-Fi networks are now widely available, the possibility of tracking people's motion paths by using the Wi-Fi received signal strength (RSS) allows the ubiquitous context-awareness and several potential innovative services [5]. For instance, if the shoppers' paths are tracked by the retailers in a store, the sales information and the related advertisements could be pushed in based on the shoppers' real-time locations [6]. As another example, the hospitals can utilize the patients' path information to identify whether they are in an emergency situation and also assign the closest doctors or nurses to see the patients, if necessary [7].

A variety of wireless network techniques have been considered for location tracking in indoor or outdoor environments. Although the popular and widely used GPS can provide accurate information for outdoor localization and path navigation services, the positioning signals are generally blocked in the indoor or underground scenarios $[8,9]$. To solve this problem, the Wi-Fi network is chosen as the favorite technique to achieve indoor localization and tracking due to the popularity in public hotspots and low cost for the deployment in practice [10-13]. In the most recent Wi-Fi localization and tracking approaches, the site-survey measurement on RSS fingerprints is required in the offline phase to construct the RSS radio map associated with the target area [14-18]. However, the adaptation degradation problem occurs due to the time consuming and labor intensive work on fingerprint recording [19]. To solve this problem, Wang et al. in [20] introduced a new idea of mapping the peoples motion paths into a mobility map in which the location points (LPs) are connected by transition relations. As discussed in [20], there are three categories of LPs involved in people's motion paths: (i) personal common locations (PCLs) which many people have spent a lot of time in, (ii) crucial locations (CLs) where 
multiple adjacent paths intersect, and (iii) ordinary locations which are used to describe the transition relations between neighboring LPs. Each LP is formed by merging the similar measurements which are recorded from assisted GPS (AGPS), Wi-Fi, and cellular networks. However, the constructed mobility map in [20] fails to consider the timestamp relations of measurements. In our previous work [21], we found that, for the calculation of measurement similarities, the timestamps and signal strengths are two sides of a coin. With this idea, we performed spectral clustering on RSS shotgun reads based on the combination of timestamps and signal strengths and also refer to Kullback-Leibler divergence of RSS distributions in different LPs to conduct mobility map construction [21]. The most significant problem to limit the practical use for the mobility map in [21] is the low precision and ambiguity in PCL identification, which means that the PCLs cannot be precisely and uniquely identified from the mobility map.

To overcome the disadvantages of the conventional approaches, we propose the tracking solution based on the time-stamped mobility map constructed from Wi-Fi RSS measurement matching in this paper. This solution complies with three basic prerequisites: (i) it can be applied to the large anonymous Wi-Fi areas by using physically unlabeled highdimensional measurements; (ii) mobility map is constructed from significant LPs which are involved in many people's motion paths; and (iii) people's motion paths are tracked in an adequate precision level. To meet these goals, we divide our approach into the following four main steps: (i) measurement quantization in a low-dimensional manifold which lies in the raw RSS space, (ii) LP identification by Smith-Waterman measurement matching [22], (iii) LP assembling into the mobility map in a temporal logic manner [23], and (iv) people's motion path tracking in mobility map.

The rest of this paper is organized as follows. Section 2 gives an overview of some relevant tracking approaches which have been used so far. In Section 3, we describe the detailed steps involved in our proposed approach. Section 4 presents the experimental results and analysis. Finally, we conclude this paper and provide some future directions in Section 5.

\section{Related Work}

As the Wi-Fi technique becomes prevalent wireless solution in public hotspots, there are a largely increasing number of different approaches used to track people's paths by using $\mathrm{Wi}$ Fi technique $[24,25]$. In general, these approaches fall into five main categories: proximity sensing, location fingerprinting, pattern matching, time trilateration, and angle triangulation.

2.1. Proximity Sensing. The proximity sensing is recognized as the simplest way to track people's locations in a real-time manner [26, 27]. The location calculation is done based on the density of access points (APs) and granularity of divided cells in target area. In most cases, the target is located at the closest cell which it most probably belongs to. In [26], the authors divided the target area into several disjoint cells and fitted the Gaussian RSS distributions for the hearable base stations from the recorded RSSs in each cell. Then, when a localization request arrives, the Bayesian probabilistic method is employed to locate the target into the cell which has the highest confidence probability. Finally, the Markov chain is used for path tracking. As another example of the proximity sensing-based location tracking, the Herecast in [27] conducted the Wi-Fi localization by using a database consisting of the APs' service set identifiers (SSIDs) and the signal coverage range of each AP. For any location request, the area corresponding to the coverage of the AP which has been detected as the strongest AP, namely, the AP associated with the largest RSS, is referred to as the receiver's estimated location. Based on this approach, it is extremely difficult to perform a finer path tracking due to the imprecise localization results.

2.2. Location Fingerprinting. The location fingerprinting has been most widely used in current location tracking systems in Wi-Fi environments [28-30]. This approach requires the constructed radio map of fingerprints. Each fingerprint is a vector of RSS associated with its physical locations which are calibrated in the offline phase. In the online phase, the target or the location server retrieves the radio map to estimate the location which has the most similar fingerprint to each newly recorded RSS measurement. The first representative RADAR system [28] was designed based on the assumption that the physically adjacent locations have the same fingerprints as in signal space. The operation of RADAR system consists of two phases. The radio map is first constructed in the offline phase to be afterwards used for location estimation. In the online phase, the target's locations are tracked by using the nearest neighbor(s) in signal space (or $K$-nearest neighbor(s) (KNN) algorithm). The Horus [30] and Nibble [29] are another two prominent fingerprint-based location tracking systems. Both the Horus and Nibble systems work based on the Bayesian inference approach, while the major difference between them is about the way to depict the RSS distributions at reference points (RPs). In Horus system, a Gaussian distribution curve for each hearable AP is fitted from the recorded RSSs at each $\mathrm{RP}$, while the Nibble system uses a histogram to record the frequencies of recorded RSSs at each RP. Moreover, from the study of the problems about RSS correlation, variations of RSSs with respect to the environmental changes, and relations of RSSs and spatial characteristics, the Horus system is featured with high accuracy and low computation cost compared to the Nibble system.

2.3. Pattern Matching. Reference [31] proposed a new location tracking system, LENSR, adopting the $K$-nearest neighborhood vector mapping-aided topological counter propagation network. Fang and Lin in [32] studied the discriminantadaptive neural network (DANN) for location tracking in Wi-Fi environment. Different from the conventional pattern matching approaches, DANN extracts the low-dimensional discriminative components for neural network training. Other similar works on pattern matching-based location tracking can be found in $[33,34]$. The pattern matching 
approach addressed in [33] relies on the multilayer perceptron architecture by one-step secant training. In [34], the pattern matching approach with well training process is proved to perform better localization accuracy than the conventional nearest neighbor(s) and Bayesian inference approaches. However, the major drawback of pattern trainingbased location tracking system is that it should be conducted by sufficient training before it works.

2.4. Trilateration and Triangulation. The basic idea of trilateration and triangulation approaches comes from the time of arrival (TOA) and angle of arrival (AOA) measurements. To enable the localization in 2-dimensional areas, the signal measurements from at least three and two APs should be made for the TOA and AOA systems, respectively [35, 36]. In TOA systems [35], the trilateration approach is conducted on the distances between the APs and tracking target which are calculated by the measured propagation time between them. Moreover, the exact time synchronization is also required for the measurement of propagation time. The main advantages of AOA systems [36] are that there are as few as two APs for the purpose of 2-dimensional localization; meanwhile, the time synchronization between the APs and tracking target is not required. However, the location precision could degrade when the signal is blocked by the walls and infrastructures or the target is located far away from the APs.

Since the TOA and AOA location systems involve significant changes on hardware devices and infrastructures which make these two systems difficult to be widely applied in practice, the RSS-based trilateration approach is more preferred by current work [37]. Different from the TOA systems, the distances between the APs and tracking target are calculated by the RSS propagation models. In [37], Narzullaev compared three representative models used for Wi-Fi RSS-based trilateration approach: (i) log-distance loss model which assumes that the mean of RSSs approximately decreases logarithmically with the propagation distance, (ii) multislope loss model which achieves a larger granularity of the predicted locations and requires a shorter sample collection time, and (iii) multiwall loss model which carefully takes the path loss caused by the walls and floors into account.

In all, applying the aforementioned location tracking approaches into the large Wi-Fi environments could be a challenging work by the reasons of the inaccurate localization results in proximity sensing, laboring cost for fingerprint calibration and training process in location fingerprinting and pattern matching, respectively, and extra devices and infrastructures required by trilateration and triangulation approaches. The main contribution of this paper is to develop a better solution to track people's motion path in large Wi-Fi environments by using RSS-based time-stamped mobility map without any fingerprint.

\section{System Description}

3.1. System Overview. Our proposed system consists of two phases: offline training phase and online tracking phase, as shown in Figure 1. The offline training phase is conducted on the network side with a large amount of computation resource, while the online tracking phase is conducted on the source-weak client side.

In the offline training phase, we first record RSS measurements to conduct measurement quantization. The quantized RSS measurements are then used to identify the raw LPs by performing the Smith-Waterman measurement matching. Each LP corresponds to a significant location which is involved in many people's motion paths. Finally, we do the LP assembling to construct the mobility map corresponding to the target area. In the online tracking phase, we first quantize each new RSS measurement into a discrete level. Then, the matching LP with respect to each new online fragment can be determined based on the fine LP matching. Finally, people's motion paths are tracked by connecting every two consecutive matching LPs along the shortest path in mobility map. For the sake of convenience, a list of notations used in this paper is given in Notation.

3.2. RSS Measurement Recording. In our system, the RSS measurements are sporadically recorded by our planned volunteers equipped with $\mathrm{Wi}$-Fi mobile receivers following their routine activities in target area. A measurement is a vector of RSS which consists of the RSS values from all the hearable APs [38]. Each string of consecutive measurements is called a fragment. As discussed in [28, 39], the measurements could be similar if they are recorded at nearby locations. We define the two fragments containing the common similar measurements as a growing fragment pair in which each overlapped piece of common similar measurements forms a raw LP to be afterwards used for LP merging and splitting to construct the time-stamped mobility map.

We set $R^{\ell}=\left\{\boldsymbol{\mu}_{1}^{\ell}, \ldots, \boldsymbol{\mu}_{N^{\ell}}^{\ell}\right\}$ as the $\ell$ th $(\ell=1, \ldots, N)$ fragment where $N$ and $N^{\ell}$ stand for the numbers of fragments and measurement in $R^{\ell}$, respectively, and $\boldsymbol{\mu}_{i}^{\ell}$ is the $i$ th $(i=$ $\left.1, \ldots, N^{\ell}\right)$ measurement. If there are $M$ hearable APs, we can obtain $\mu_{i}^{\ell}=\left(\mu_{i, 1}^{\ell}, \ldots, \mu_{i, M}^{\ell}\right)$, where $\mu_{i, j}^{\ell}(j=1, \ldots, M)$ is the RSS value from AP $j$. In each growing fragment pair (i.e., $\left.\left\{R^{s}, R^{t}\right\}(s, t \in\{1, \ldots, N\})\right)$, the $k$ th $\left(k=1, \ldots, N_{s, t}\right)$ LP is denoted as $P_{s, t}^{k}$. After all the LPs are obtained, the mobility map we seek to construct is recognized as a graph $G=$ $\left(V_{P}, E_{P}\right)$ in which $V_{P}$ and $E_{P}$ stand for the sets of LPs and timestamped transition relations between neighboring LPs, as previously discussed in [21].

3.3. Measurement Quantization. For the sake of applying Smith-Waterman measurement matching technique to construct mobility map, we need to quantize the RSS measurements into different discrete levels based on the similarities of RSS measurements. Specifically, we use Laplacian embedding-based spectral clustering to quantize the RSS measurements which have been merged into the same cluster in the same quantization level. Thus, the number of clusters by spectral clustering equals the number of quantization levels. The detailed steps of measurement quantization process are provided as follows. 


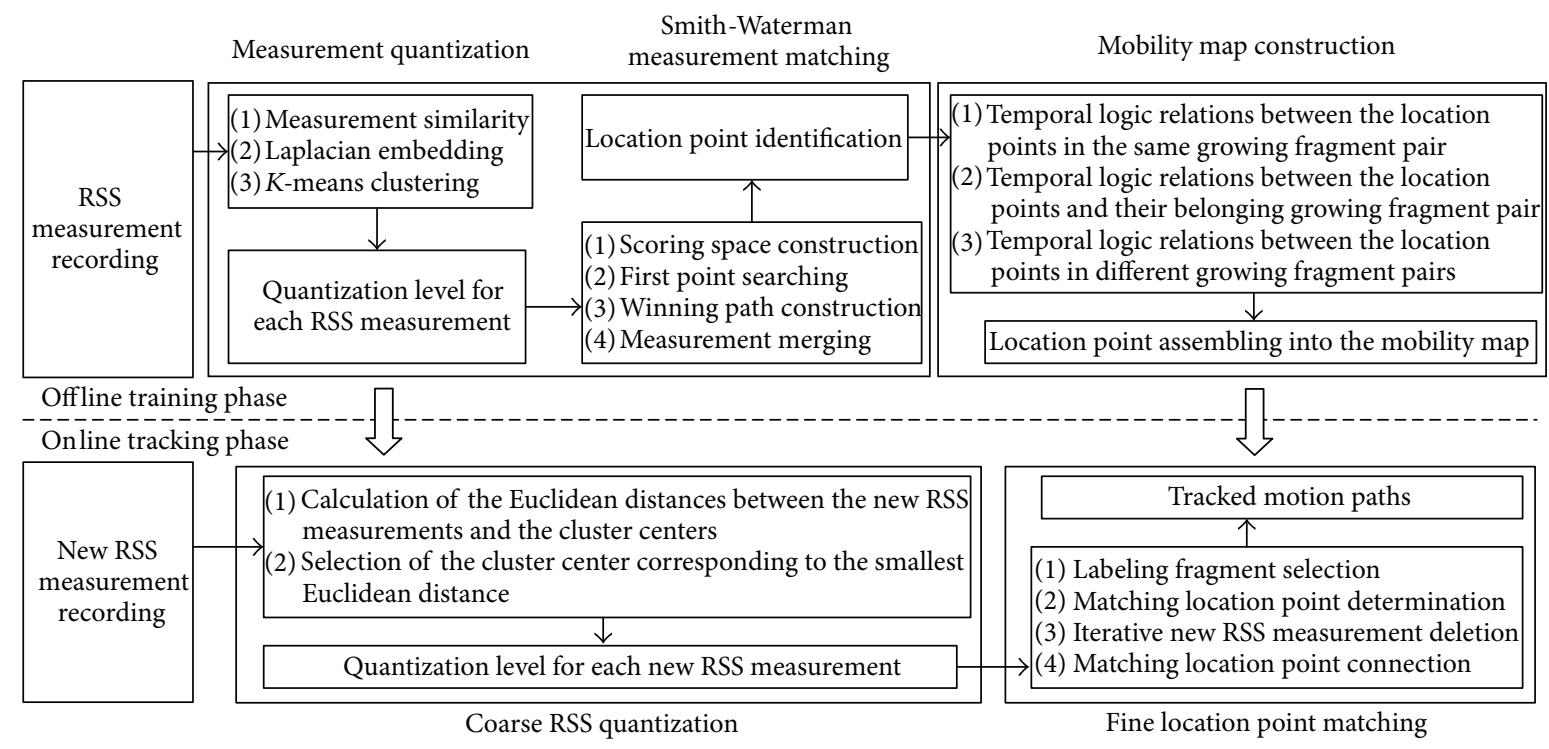

FIGURE 1: Architecture of the proposed system.

Step 1. We calculate the similarity of $\boldsymbol{\mu}_{i}^{\ell}$ and $\boldsymbol{\mu}_{i^{\prime}}^{\ell^{\prime}}\left(i^{\prime}=1, \ldots\right.$, $\left.N^{\ell} ; \ell^{\prime}=1, \ldots, N\right)$ as $W_{i, i^{\prime}}^{\ell, \ell^{\prime}}=\exp \left(-\operatorname{diff}_{R}\left(\boldsymbol{\mu}_{i}^{\ell}, \boldsymbol{\mu}_{i^{\prime}}^{\ell^{\prime}}\right)\right)$ where $\operatorname{diff}_{R}\left(\boldsymbol{\mu}_{i}^{\ell}, \boldsymbol{\mu}_{i^{\prime}}^{\ell^{\prime}}\right)=\left\|\boldsymbol{\mu}_{i}^{\ell}-\boldsymbol{\mu}_{i^{\prime}}^{\ell^{\prime}}\right\|_{2} / \max \left\{\left\|\boldsymbol{\mu}_{i}^{\ell}-\boldsymbol{\mu}_{i^{\prime}}^{\ell^{\prime}}\right\|_{2}\right\}$.

Step 2. Considering the problem of mapping the raw measurements into a $K$-dimensional $(K<M)$ space, we can represent the mapped measurements as a $\left(\sum_{\ell=1}^{N} N^{\ell}\right) \times K$ matrix $\Psi=\left[\widehat{\boldsymbol{\mu}}_{1}^{1} \cdots \widehat{\boldsymbol{\mu}}_{N^{1}}^{1} \cdots \widehat{\boldsymbol{\mu}}_{1}^{\ell} \cdots \widehat{\boldsymbol{\mu}}_{N^{\ell}}^{\ell} \cdots \widehat{\boldsymbol{\mu}}_{1}^{N} \cdots \widehat{\boldsymbol{\mu}}_{N^{N}}^{N}\right]^{\mathrm{T}}$, where the superscript " $T$ " denotes the transpose operation and $\hat{\boldsymbol{\mu}}_{i}^{\ell}=$ $\left(r_{1, i}^{\ell}, \ldots, r_{K, i}^{\ell}\right)$ denotes the mapped vector of $\boldsymbol{\mu}_{i}^{\ell}$. Based on the Laplacian embedding [40], we can obtain the optimal objective function as

$$
\begin{gathered}
\min _{\Psi}\left\{\sum_{\ell, \ell^{\prime}=1}^{N} \sum_{i, i^{\prime}=1}^{N^{\ell}} \operatorname{diff}_{R}^{2}\left(\widehat{\boldsymbol{\mu}}_{i}^{\ell}, \widehat{\boldsymbol{\mu}}_{i^{\prime}}^{\ell^{\prime}}\right) W_{i, i^{\prime}}^{\ell, \ell^{\prime}}\right\} \\
=\min _{\Psi}\left\{\operatorname{tr}\left((\Psi)^{T}(\mathbf{D}-\mathbf{W}) \Psi\right)\right\},
\end{gathered}
$$

where "tr" denotes the trace operation, $\mathbf{D}=\left[D_{i, i^{\prime}}^{\ell, \ell^{\prime}}\right]_{i, i^{\prime}=1 ; \ell, \ell^{\prime}=1}^{N^{\ell}}$, and $\mathbf{W}=\left[W_{i, i^{\prime}}^{\ell, \ell^{\prime}}\right]_{i, i^{\prime}=1 ; \ell, \ell^{\prime}=1}^{N^{\ell} ; N}$. When $i=i^{\prime}$ and $\ell=\ell^{\prime}$, we have $D_{i, i^{\prime}}^{\ell, \ell^{\prime}}=\sum_{\ell^{\prime}=1}^{N} \sum_{i^{\prime}=1}^{N_{\ell^{\prime}}} W_{i, i^{\prime}}^{\ell, \ell^{\prime}}$; otherwise, we have $D_{i, i^{\prime}}^{\ell, \ell^{\prime}}=0$. As discussed in [21], the solution to the optimization problem in (1) can be given by the $K$ eigenvectors associated with the smallest eigenvalues of the eigenvalue problem in (2):

$$
\begin{array}{ll}
\text { minimize } & \sum_{\gamma=1}^{K} \lambda_{\gamma} \\
\text { subject to } & \mathbf{L} \hat{\boldsymbol{\mu}}_{i}^{\ell}=\lambda_{\gamma} \mathbf{D} \hat{\boldsymbol{\mu}}_{i}^{\ell}, \\
& i=1, \ldots, N^{\ell} ; \quad \ell=1, \ldots, N ; \quad \gamma=1, \ldots, K .
\end{array}
$$

Step 3. We perform $K$-means clustering on the mapped $K$ dimensional vectors to obtain the $\Phi$ clusters, $C_{1}, \ldots, C_{\Phi}$, where $C_{\omega}$ denotes the $\omega$ th $(\omega=1, \ldots, \Phi)$ cluster. Then, we quantize the RSS measurements corresponding to the mapped vectors in the same cluster into the same quantization level.

3.4. Smith-Waterman Measurement Matching. The objective of Smith-Waterman measurement matching is to identify the raw LPs for the construction of mobility map associated with the target area. To meet this goal, we adopt the SmithWaterman alignment approach to find the winning paths in the scoring space for each growing fragment pair and then perform measurement matching to identify the raw LPs. The steps of the raw LP identification are as follows.

Step 1. In growing fragment pair $\left\{R^{s}, R^{t}\right\}\left(R^{s}=\left\{\boldsymbol{\mu}_{1}^{s}, \ldots, \boldsymbol{\mu}_{N^{s}}^{s}\right\}\right.$, $\left.R^{t}=\left\{\boldsymbol{\mu}_{1}^{t}, \ldots, \boldsymbol{\mu}_{N^{t}}^{t}\right\}\right)$, when $\boldsymbol{\mu}_{p}^{s}\left(p \in\left\{1, \ldots, N^{s}\right\}\right)$ and $\boldsymbol{\mu}_{q}^{t}(q \in$ $\left.\left\{1, \ldots, N^{t}\right\}\right)$ are in the same quantization level, we set a positive matching score, $\varphi\left(\boldsymbol{\mu}_{p}^{s}, \boldsymbol{\mu}_{q}^{t}\right)$, for the measurement pair $\left(\boldsymbol{\mu}_{p}^{s}, \boldsymbol{\mu}_{q}^{t}\right)$; otherwise, we set a negative mismatching score, 
$\psi\left(\boldsymbol{\mu}_{p}^{s}, \boldsymbol{\mu}_{q}^{t}\right)$, for $\left(\boldsymbol{\mu}_{p}^{s}, \boldsymbol{\mu}_{q}^{t}\right)$. The negative missing scores, $\phi_{w}\left(\boldsymbol{\mu}_{p}^{s},-\right)$ and $\phi_{w}\left(-, \boldsymbol{\mu}_{q}^{t}\right)$, are set when there is no measurement in $R^{t}$ and $R^{s}$ matched with $\boldsymbol{\mu}_{p}^{s}$ and $\boldsymbol{\mu}_{q}^{t}$, respectively. We have the relations of "matching score $>0>$ missing score $>$ mismatching score." Then, we can obtain the scoring space, $\mathbf{H}_{s, t}=\left[H\left(\boldsymbol{\mu}_{p}^{s}, \boldsymbol{\mu}_{q}^{t}\right)\right]_{p=1 ; q=1}^{N^{s} ; N^{t}}$, for the growing fragment pair $\left\{R^{s}, R^{t}\right\}$, as shown in the following:

$$
\begin{aligned}
& \mathbf{H}_{s, t}= {\left[H\left(\boldsymbol{\mu}_{p}^{s}, \boldsymbol{\mu}_{q}^{t}\right)\right]_{p=1 ; q=1}^{N^{s} ; N^{t}} } \\
&= {\left[\max \left\{\begin{array}{c}
H\left(\boldsymbol{\mu}_{p-1}^{s}, \boldsymbol{\mu}_{q-1}^{t}\right)+\varphi\left(\boldsymbol{\mu}_{p}^{s}, \boldsymbol{\mu}_{q}^{t}\right), \boldsymbol{\mu}_{p}^{s} \text { and } \boldsymbol{\mu}_{q}^{t} \text { are in the same level; } \\
H\left(\boldsymbol{\mu}_{p-1}^{s}, \boldsymbol{\mu}_{q-1}^{t}\right)+\psi\left(\boldsymbol{\mu}_{p}^{s}, \boldsymbol{\mu}_{q}^{t}\right), \boldsymbol{\mu}_{p}^{s} \text { and } \boldsymbol{\mu}_{q}^{t} \text { are in different levels; } \\
\max _{w=1}^{p-1}\left\{H\left(\boldsymbol{\mu}_{p-w}^{s}, \boldsymbol{\mu}_{q}^{t}\right)+\phi_{w}\left(\boldsymbol{\mu}_{p}^{s},-\right)\right\} ; \\
\max _{w=1}^{-1}\left\{H\left(\boldsymbol{\mu}_{p}^{s}, \boldsymbol{\mu}_{q-w}^{t}\right)+\phi_{w}\left(-, \boldsymbol{\mu}_{q}^{t}\right)\right\} ; \\
0
\end{array}\right\}\right]_{p=1 ; q=1}^{N^{s} ; N^{t}} } \\
& H\left(\boldsymbol{\mu}_{p^{\prime}}^{s}, \boldsymbol{\mu}_{0}^{t}\right)=0, p^{\prime}=1, \ldots, N^{s}, \\
& H\left(\boldsymbol{\mu}_{0}^{s}, \boldsymbol{\mu}_{q^{\prime}}^{t}\right)=0, q^{\prime}=1, \ldots, N^{t} .
\end{aligned},
$$

Step 2. We select the measurement pair, $\left(\boldsymbol{\mu}_{\tilde{p}}^{s}, \boldsymbol{\mu}_{\tilde{q}}^{t}\right)$, which has the highest score in scoring space as the first point on the winning path, such that $\left(\boldsymbol{\mu}_{\tilde{p}}^{s}, \boldsymbol{\mu}_{\tilde{q}}^{t}\right)=\arg \max _{p=1 ; q=1}^{N^{s} ; N^{t}}\left\{H\left(\boldsymbol{\mu}_{p}^{s}, \boldsymbol{\mu}_{q}^{t}\right)\right\}$. We require that the score of the first point should be higher than $\eta_{M}$ (i.e., $H\left(\boldsymbol{\mu}_{\tilde{p}}^{s}, \boldsymbol{\mu}_{\tilde{q}}^{t}\right)>\eta_{M}$ ), where $\eta_{M}$ is the threshold for measurement matching. In our experiments, we set $\eta_{M}=30$.

Step 3. We compare the scores of three previous measurement pairs, $\left(\boldsymbol{\mu}_{\tilde{p}-1}^{s}, \boldsymbol{\mu}_{\tilde{q}-1}^{t}\right),\left(\boldsymbol{\mu}_{\tilde{p}-1}^{s}, \boldsymbol{\mu}_{\tilde{q}}^{t}\right)$, and $\left(\boldsymbol{\mu}_{\tilde{p}}^{s}, \boldsymbol{\mu}_{\tilde{q}-1}^{t}\right)$, and select the pair which has the highest score among them as the second point on the winning path. We repeat this process until the selected pair has the score zero. At this point, the selected pair with the score zero is defined as the last point on the winning path.

Step 4. After the winning path in scoring space is obtained, we identify the corresponding raw LP by merging the matched measurement pairs. Based on the Smith-Waterman alignment, the three measurement matching criteria are provided as follows.

(i) Criterion 1: measurements $\boldsymbol{\mu}_{p}^{s}$ and $\boldsymbol{\mu}_{q}^{t}$ are matched when there is a diagonal jump from $\left(\boldsymbol{\mu}_{p-1}^{s}, \boldsymbol{\mu}_{q-1}^{t}\right)$ to $\left(\boldsymbol{\mu}_{p}^{s}, \boldsymbol{\mu}_{q}^{t}\right)$ in scoring space.

(ii) Criterion 2: measurement $\mu_{p}^{s}$ is not matched with any measurement in fragment $R^{t}$ when there is a topdown jump from $\left(\boldsymbol{\mu}_{p-1}^{s}, \boldsymbol{\mu}_{q}^{t}\right)$ to $\left(\boldsymbol{\mu}_{p}^{s}, \boldsymbol{\mu}_{q}^{t}\right)$ in scoring space.

(iii) Criterion 3: measurement $\mu_{q}^{t}$ is not matched with any measurement in fragment $R^{s}$ when there is a left-right jump from $\left(\boldsymbol{\mu}_{p}^{s}, \boldsymbol{\mu}_{q-1}^{t}\right)$ to $\left(\boldsymbol{\mu}_{p}^{s}, \boldsymbol{\mu}_{q}^{t}\right)$ in scoring space.

To identify the other raw LPs from the scoring space, we continue to select the measurement pair which has the highest score in the remaining measurement pairs which are not involved in the previous winning paths as the first point of a new winning path. We follow Steps 3 and 4 until this new winning path arrives at a measurement pair which has the score zero or is involved in the previous winning paths. We name this measurement pair as the last point on this new winning path. For simplicity, we only focus on the situation that only one raw LP exists in a scoring space (i.e., $N_{s, t}=1$ for the growing fragment pair $\left\{R^{s}, R^{t}\right\}$ ) since the situation of multiple raw LPs can be avoided by manually chopping each longlength fragment into several shorter ones. The length of a fragment is defined as the number of measurements contained in this fragment.

3.5. Mobility Map Construction. After all the raw LPs have been identified, the next work is to assemble the raw LPs into the mobility map in a temporal logic manner. As discussed before, since the measurements in each raw LP are labeled by timestamps, we can approximately represent each raw LP as a time interval which starts at the last point and ends at the first point on its corresponding winning path. Then, the raw LP assembling process can be converted into a temporal reasoning problem, as introduced in [23]. The detailed steps are described below.

Step 1. Based on Allen's interval algebra (i.e., 13 temporal logic relations: $\{=\},\{\mathrm{m}\},\{\mathrm{mi}\},\{\mathrm{o}\},\{\mathrm{oi}\},\{\mathrm{s}\},\{\mathrm{si}\},\{\mathrm{f}\},\{\mathrm{fi}\},\{\mathrm{d}\},\{\mathrm{di}\},\{<\}$, and $\{>\})$ in [23], we can capture the temporal logic relations between the raw LPs in each growing fragment pair. Specifically, when $P_{s, t}^{k}$ and $P_{s, t}^{k^{\prime}}$ are two raw LPs for the growing fragment pair $\left\{R^{s}, R^{t}\right\}$, we obtain the following:

(i) if the last point in $P_{s, t}^{k^{\prime}}$ is located in $P_{s, t}^{k}$, we have $P_{s, t}^{k}\{\mathrm{~m}\} P_{s, t}^{k^{\prime}}$; 
(ii) if the last point in $P_{s, t}^{k}$ is located in $P_{s, t}^{k^{\prime}}$, we have $P_{s, t}^{k}\{\mathrm{mi}\} P_{s, t}^{k^{\prime}}$;

(iii) if the last point in $P_{s, t}^{k^{\prime}}$ is after the first point in $P_{s, t}^{k}$, we have $P_{s, t}^{k}\{<\} P_{s, t}^{k^{\prime}}$;

(iv) if the last point in $P_{s, t}^{k}$ is after the first point in $P_{s, t}^{k^{\prime}}$, we have $P_{s, t}^{k}\{>\} P_{s, t}^{k^{\prime}}$.

In (4), when the timestamp of the last point in a raw LP is larger than the timestamp of the first point of another raw LP, we define "the last point is after the first point"; otherwise, we define "the last point is before the first point."

Step 2. The temporal logic relations between the raw LPs (i.e., $\left.P_{s, t}^{k}\right)$ and their belonging growing fragment pair (i.e., $\left\{R^{s}, R^{t}\right\}$ ) are given as follows:

(i) if the start point in $R^{s}$ (or $R^{t}$ ) is located in $P_{s, t}^{k}$ and the end point in $R^{s}$ (or $R^{t}$ ) is after the first point in $P_{s, t}^{k}$, we have $P_{s, t}^{k}\{s\} R^{s}$ (or $\left.R^{t}\right)$;

(ii) if the start point in $R^{s}$ (or $R^{t}$ ) is before the last point in $P_{s, t}^{k}$ and the end point in $R^{s}\left(\right.$ or $\left.R^{t}\right)$ is after the first point in $P_{s, t}^{k}$, we have $P_{s, t}^{k}\{\mathrm{~d}\} R^{s}\left(\right.$ or $\left.R^{t}\right)$;

(iii) if the start point in $R^{s}$ (or $R^{t}$ ) is before the last point in $P_{s, t}^{k}$ and the end point in $R^{s}$ (or $R^{t}$ ) is located in $P_{s, t}^{k}$, we have $P_{s, t}^{k}\{\mathrm{f}\} R^{s}\left(\right.$ or $\left.R^{t}\right)$;

(iv) if both the start and end points in $R^{s}$ (or $R^{t}$ ) are located in $P_{s, t}^{k}$, we have $P_{s, t}^{k}\{=\} R^{s}\left(\right.$ or $\left.R^{t}\right)$,

where the start and end points in $R^{s}$ (or $R^{t}$ ) are defined as the measurements which have the smallest and largest timestamps in $R^{s}$ (or $R^{t}$ ), respectively.

Step 3. Since the mobility map we seek to construct is a connected graph, the temporal logic relations of any two raw LPs can be obtained by Allen's interval algebra based on the timestamped transitions between the LPs and fragments. To illustrate this result clearer, we use the transitivity table in [23] to show the temporal logic relations between the different raw LPs. Table 1 gives the possible temporal logic relations between any two LPs (i.e., $P_{s, t}^{k}$ and $P_{t, u}^{k^{\prime}}$ ) belonging to the two different growing fragment pairs (i.e., $\left\{R^{s}, R^{t}\right\}$ and $\left\{R^{t}, R^{u}\right\}$ ).
We take the relations of $P_{s, t}^{k}\{s\} R^{t}$ and $P_{t, u}^{k^{\prime}}\{s\} R^{t}$, for instance. Based on the transitivity table, there are three possible temporal logic relations between $P_{s, t}^{k}$ and $P_{t, u}^{k^{\prime}}$ (i.e., $P_{s, t}^{k}\{s\} P_{t, u}^{k^{\prime}}$, $P_{s, t}^{k}\{\operatorname{si}\} P_{t, u}^{k^{\prime}}$, and $\left.P_{s, t}^{k}\{=\} P_{t, u}^{k^{\prime}}\right)$, such that

(i) if the first point in $P_{t, u}^{k^{\prime}}$ is after the first point in $P_{s, t}^{k}$, we have $P_{s, t}^{k}\{s\} P_{t, u}^{k^{\prime}}$;

(ii) if the first point in $P_{s, t}^{k}$ is after the first point in $P_{t, u}^{k^{\prime}}$, we have $P_{s, t}^{k}\{\mathrm{si}\} P_{t, u}^{k^{\prime}}$;

(iii) if the first points in $P_{s, t}^{k}$ and $P_{t, u}^{k^{\prime}}$ are the same, we have $P_{s, t}^{k}\{=\} P_{t, u}^{k^{\prime}}$.

Finally, the block diagram for the LP assembling into a mobility map is shown in Figure 2. We also take the relations of $P_{s, t}^{k}\{s\} R^{t}$ and $P_{t, u}^{k^{\prime}}\{s\} R^{t}$, for instance. Based on (6) and Figure 2, (i) if the first points in $P_{s, t}^{k}$ and $P_{t, u}^{k^{\prime}}$ are the same (i.e., $\left.R_{P}\left(P_{s, t}^{k}, P_{t, u}^{k^{\prime}}\right)=\{=\}\right)$, we merge $P_{t, u}^{k^{\prime}}$ into $P_{s, t}^{k}$ to form a new LP consisting of all the measurement pairs in $P_{s, t}^{k}$ and $P_{t, u}^{k^{\prime}}$; (ii) if the first point in $P_{t, u}^{k^{\prime}}$ is after the first point in $P_{s, t}^{k}$ (i.e., $\left.R_{P}\left(P_{s, t}^{k}, P_{t, u}^{k^{\prime}}\right)=\{s\}\right)$, we merge all the overlapped measurement pairs in $P_{t, u}^{k^{\prime}}$ into $P_{s, t}^{k}$ and then delete all the overlapped measurement pairs in $P_{t, u}^{k^{\prime}}$; and (iii) if the first point in $P_{s, t}^{k}$ is after the first point in $P_{t, u}^{k^{\prime}}$ (i.e., $R_{P}\left(P_{s, t}^{k}, P_{t, u}^{k^{\prime}}\right)=\{$ si $\}$ ), we merge all the overlapped measurement pairs in $P_{s, t}^{k}$ into $P_{t, u}^{k^{\prime}}$ and then delete all the overlapped measurement pairs in $P_{s, t}^{k}$.

3.6. Path Tracking in Mobility Map. There are two main steps involved in path tracking: (i) coarse RSS quantization and (ii) fine LP matching. The path tracking in mobility map is conducted as follows.

Step 1 (coarse RSS quantization). As discussed in Section 3.2, after the offline RSS measurement quantization, we can obtain $\Phi$ clusters associated with the $\Phi$ quantization levels. Then, for each new measurement, $\boldsymbol{\mu}_{\tau}^{\mathrm{New}}\left(\tau=1, \ldots, N^{\mathrm{New}}\right)$, in the online fragment, $R^{\text {New }}=\left\{\boldsymbol{\mu}_{1}^{\text {New }}, \ldots, \boldsymbol{\mu}_{N^{\text {New }}}^{\text {New }}\right\}$, where $N^{\text {New }}$ is the number of new measurements in $R^{\mathrm{New}}$, we calculate the Euclidean distance between $\boldsymbol{\mu}_{\tau}^{\mathrm{New}}$ and the average measurement in each cluster (i.e., $\operatorname{Avg}\left(C_{\omega}\right)(\omega=1, \ldots, \Phi)$ ), $\operatorname{diff}_{R}\left(\boldsymbol{\mu}_{\tau}^{\text {New }}, \operatorname{Avg}\left(C_{\omega}\right)\right)$, and then quantize the new measurement in a discrete level of cluster $C_{\widehat{\omega}}$, such that

$$
\widehat{\omega}=\underset{\omega=1, \ldots, \Phi}{\arg \min }\left\{\operatorname{diff}_{R}\left(\boldsymbol{\mu}_{\tau}^{\mathrm{New}}, \operatorname{Avg}\left(C_{\omega}\right)\right)\right\} .
$$

Step 2 (fine LP matching). We select the fragment which has the longest length in each LP as the labeling 
TABLE 1: Transitivity table for different growing fragment pairs.

\begin{tabular}{lccc}
\hline$R_{p}\left(P_{s, t}^{k}, P_{t, u}^{k^{\prime}}\right)$ & $P_{t, u}^{k^{\prime}}\{\mathrm{s}\} R^{t}$ & $P_{t, u}^{k^{\prime}}\{\mathrm{d}\} R^{t}$ & $P_{t, u}^{k^{\prime}}\{\mathrm{f}\} R^{t}$ \\
\hline$P_{s, t}^{k}\{\mathrm{~s}\} R^{t}$ & $\{\mathrm{~s}, \mathrm{si},=\}$ & $\{<, \mathrm{o}, \mathrm{m}, \mathrm{di}, \mathrm{fi}\}$ & $\{<, \mathrm{o}, \mathrm{m}\}$ \\
$P_{s, t}^{k}\{\mathrm{~d}\} R^{t}$ & $\{>, \mathrm{oi}, \mathrm{mi}, \mathrm{d}, \mathrm{f}\}$ & No-info & $\{<, \mathrm{o}, \mathrm{m}, \mathrm{d}, \mathrm{s}\}$ \\
$P_{s, t}^{k}\{\mathrm{f}\} R^{t}$ & $\{>, \mathrm{oi}, \mathrm{mi}\}$ & $\{>, \mathrm{oi}, \mathrm{mi}, \mathrm{di}, \mathrm{si}\}$ & $\{<, \mathrm{o}, \mathrm{m}, \mathrm{d}, \mathrm{s}\}$ \\
$P_{s, t}^{k}\{=\} R^{t}$ & $\{\mathrm{si}\}$ & $\{\mathrm{di}\}$ & $\{\mathrm{fi}\}$
\end{tabular}

"No-info" means that all the temporal logic relations are applied.

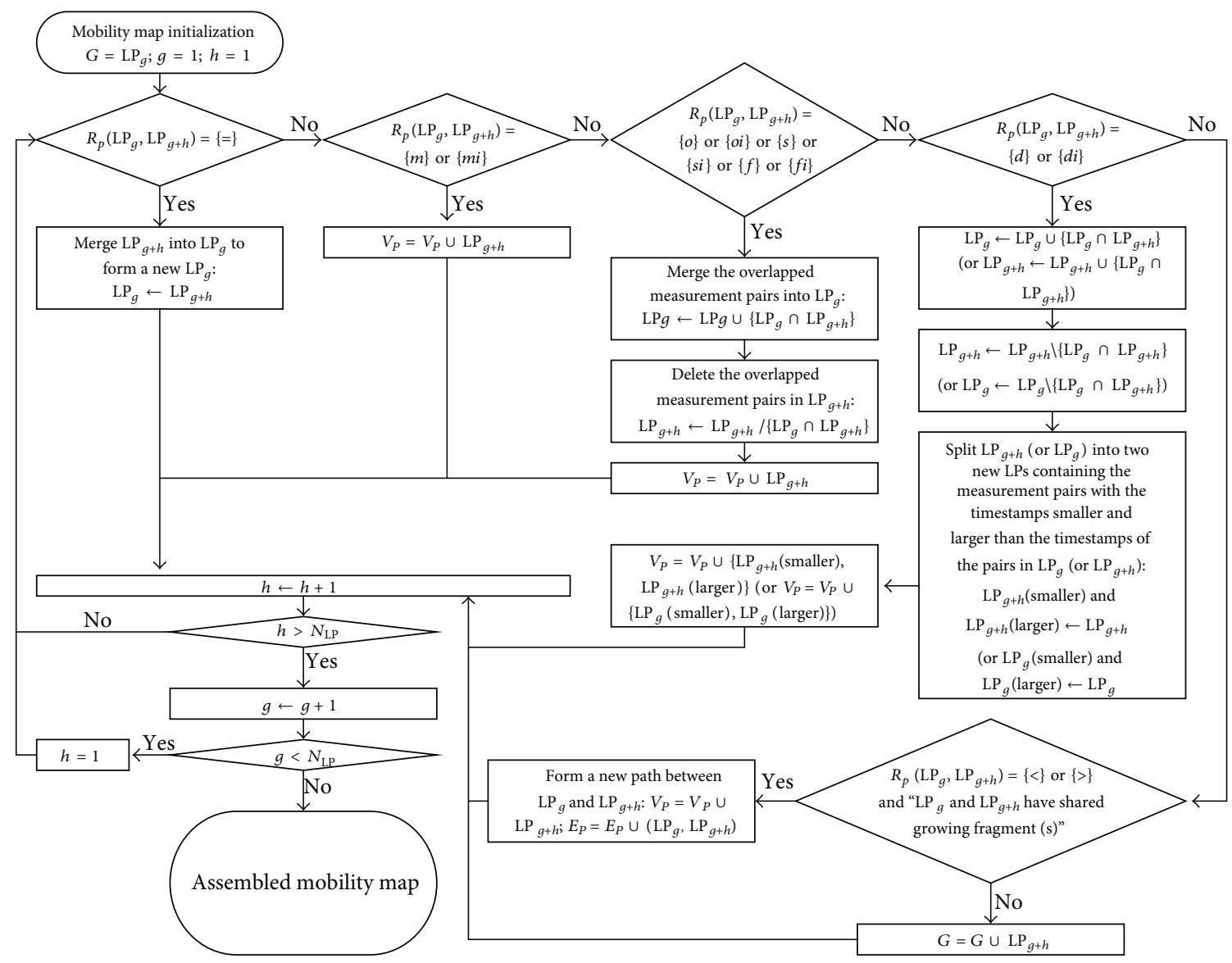

FIGURE 2: Block diagram for the LP assembling into a mobility map.

fragment. The labeling fragment in $C_{\omega}$ is denoted as $R_{\omega}^{\ell}=\left\{\boldsymbol{\mu}_{1}^{\ell}, \ldots, \boldsymbol{\mu}_{N_{\omega}^{\ell}}^{\ell}\right\}$, where $N_{\omega}^{\ell}$ is the number of measurements contained in $R_{\omega}^{\ell}$. When the labeling fragment $R_{\omega^{*}}^{\ell}$ satisfies the relation of

$$
\begin{aligned}
& \left(\omega^{*}, \tau^{*}\right) \\
& =\underset{\omega=1, \ldots, \Phi ; \tau=1, \ldots, N^{\text {New }}}{\arg \max }\left\{\max _{v=1, \ldots, N_{\omega}^{\ell}}\left\{H\left(\boldsymbol{\mu}_{v}^{\ell}, \boldsymbol{\mu}_{\tau}^{\mathrm{New}}\right)\right\}\right\},
\end{aligned}
$$

we set $C_{\omega^{*}}$ as the matching LP. After that, all the new measurements before $\boldsymbol{\mu}_{\tau^{*}}^{\mathrm{New}}$ (or the new measurements with IDs not larger than $\tau^{*}$ ) are deleted to form a new online fragment (i.e., $\left.R^{\text {New }} \leftarrow R^{\text {New }} \backslash\left\{\boldsymbol{\mu}_{\tau^{\prime}}^{\text {New }}\left(\tau^{\prime} \leq \tau^{*}\right)\right\}\right)$ and then continue to search for the next matching LP. We repeat this process until
TABLE 2: Time cost for spectral clustering.

\begin{tabular}{lccc} 
Number of measurements & & & \\
& Test 1 & Test 2 & Test 3 \\
\hline 100 & 0.36 & 0.42 & 0.41 \\
200 & 2.25 & 2.16 & 2.22 \\
300 & 7.83 & 8.28 & 8.06 \\
400 & 20.63 & 20.75 & 20.62 \\
500 & 46.49 & 46.56 & 46.88 \\
600 & 111.46 & 110.02 & 110.34 \\
\hline
\end{tabular}


TABLE 3: Fragment representation by amino acids.

\begin{tabular}{lc}
\hline Fragment IDs & Representation \\
\hline$R^{1}$ (with the length of 191) & HHHKKKKKKKIKKKKKKKKKKKKHHHHKKHHHHHHKKIILLIILLDDLL \\
& IIKKIIILLLLDDIIKKIIIIIIKKKKKHHHHHKKIIDYSLDDLDDLDLILLLIID \\
DSSSSLLLLIISSDDLLLLDDYYYYSSYYYYCCYYYYCCCCYYSSCCYYY & YLLYYCCCCYYYYYYDDDDSSYYSSDDSSLLYYY \\
\hline & RRAAGGVVQQQQQQPPWWFFWWQQVVPPFFFFEEFFEEEEEEEEEEEEE \\
$R^{2}$ (with the length of 195) & EEEFFFFEEEEEEEFFFFWWWWWWPPPPQQQQQQVNNGGGGRAANAN \\
& NNTTTTTTTTTTMMMMMMMMMMMMMMMHHHHHHKKIILLDDSSSS \\
& SSLICCCCCCCSCCCCCCCCCSCCYYYYCCYYCCYYSSLLDDLLDDDDDD \\
$R^{3}$ (with the length of 85$)$ & LS \\
\hline$R^{4}$ (with the length of 106) & AARRGGQQQQPPQQPPWWWWPPQQQQWWFFFFFFEEEEEEEEEEEEEEE \\
\hline$R^{5}$ (with the length of 80$)$ & GGRGGVQQPPQPPPWWWFEEEEFEFFWWPPPPWWPPPQPPPPPPPPPPPPP \\
\hline
\end{tabular}

there is no new measurement which remains in the online fragment or the score for the newly formed fragment is lower than the threshold, $\varepsilon_{S}$. In our experiments, we set $\varepsilon_{S}=10$. After all the matching LPs are obtained, we track the people's motion paths by connecting every two consecutive matching LPs along the shortest path in mobility map. The shortest path is defined as the path which passes by the smallest number of LPs.

Some of the raw RSS fragments recorded may be very long. When the number of RSS measurements in a fragment is too long, the computation problem may arise for the process of LP assembling into a mobility map, while the main computation cost is involved in the offline training phase. In the online tracking phase, when the user sends a location query with its new RSS fragment, our system retrieves the cluster centers and returns the quantization level as well as the highest score in scoring space. The LP corresponding to the highest score is selected as the matching LP. At this point, the calculation complexity when our system tracks hundreds and thousands of people walking around in the target area forms an interesting work in future. To clearly show the computation cost required in offline training phase, we take the spectral clustering, for example. By using the MATLAB 7.10.0 (R2010a) under WINDOWS XP system, Table 2 compares the time cost for spectral clustering in different numbers of measurements conditions. All the computations are run on a PC with Intel Core i3-2120 CPU. In Table 2, we can find that as the number of measurements increases, the time cost for spectral clustering will also increase.

\section{Experimental Results and Analysis}

In this section, we will evaluate the performance of mobility map construction and motion path tracking based on the actual RSS fragments (of dimensions 650) recorded on five representative paths in HKUST campus. The five fragments are recorded on path $1, R^{1}=\left(R^{11}, R^{12}\right)$, which is from North
Bus Stop to Library and with the length of 191; path 2, $R^{2}=$ $\left(R^{21}, R^{22}, R^{23}, R^{24}\right)$, which is from Lab 2149 to Library and with the length of 195; path $3, R^{3}=\left(R^{31}, R^{32}\right)$, which is from Lab 2149 to Coffee Shop and with the length of 85 ; path $4, R^{4}=$ $\left(R^{41}, R^{42}\right)$, which is from Lab 2149 to Office 2514 and with the length of 106; and path $5, R^{5}=\left(R^{51}, R^{52}\right)$, which is from Lab 2149 to LT-J theater and with the length of 80 [41]. Each path consists of several physically adjacent traces. We take the fragment recorded on path 1 (i.e., $R^{1}$ ), for instance. $R^{1}$ contains two consecutive segments, $R^{11}$ and $R^{12}$, which are recorded on trace 1 (between North Bus Stop and Atrium) and trace 2 (between Atrium and Library), respectively. The traces labeled by superscript “*” (i.e., trace 2 , trace 6, and trace 7 ) are the LPs to be assembled in our testing. The notation " $\rightarrow$ " indicates the path direction. A summary of these fragments and the corresponding traces is shown in Figure 3.

First of all, based on the raw measurement space consisting of 657 measurements with dimensions of 650 in Figure 4, we can calculate the similarity of any two measurements in Figure 5 . The large similarity values (in range of $[0,1]$ ) represent that the corresponding measurement pairs are extremely similar. Moreover, the largest similarity value (or value 1) can be achieved by the similarity between any measurement and itself as expected.

As discussed in Section 3.2, the optimization problem in (1) can be converted into the generalized eigenvalue problem in (2). Figure 6 shows the three eigenvectors (of dimensions 657) associated with the $K=3$ smallest eigenvalues. Since the first eigenvector is a constant vector with the eigenvalue zero, we only use the second and third eigenvectors associated with the eigenvalues 0.91 and 0.94 , respectively, as the basis of the mapped two-dimensional space. Then, we obtain the 20 clusters (or quantization levels) in the mapped two-dimensional space in Figure 7. For the Smith-Waterman measurement matching, the 20 quantization levels can be recognized as the 20 amino acids. We denote the 20 amino acids as follows: Cys (C), Ser (S), Thr (T), Pro (P), Ala (A), Gly (G), Asn (N), Asp (D), Glu (E), Gln (Q), His (H), Arg (R), Lys (K), 


\begin{tabular}{|c|c|c|c|c|c|c|c|}
\hline $\begin{array}{l}\text { Tragment IDs } \\
\text { IDs }\end{array}$ & $\begin{array}{l}\text { Trace } 1 \\
\text { between } \\
\text { north bus } \\
\text { stop and } \\
\text { atrium }\end{array}$ & $\begin{array}{l}\text { Trace } 2^{*} \\
\text { between } \\
\text { atrium and } \\
\text { library }\end{array}$ & $\begin{array}{l}\text { Trace } 3 \\
\text { between } \\
\text { atrium and } \\
\text { coffee } \\
\text { shop }\end{array}$ & $\begin{array}{l}\text { Trace } 4 \\
\text { between } \\
\text { coffee } \\
\text { shop and } \\
\text { office } 2514\end{array}$ & $\begin{array}{l}\text { Trace } 5 \\
\text { between } \\
\text { elevator } \\
\text { and LT-J } \\
\text { theater }\end{array}$ & $\begin{array}{l}\text { Trace } 6{ }^{*} \\
\text { between } \\
\text { coffee } \\
\text { shop and } \\
\text { elevator }\end{array}$ & $\begin{array}{l}\text { Trace } 7^{*} \\
\text { between } \\
\text { elevator } \\
\text { and Lab } \\
2149 \\
\end{array}$ \\
\hline$R^{1}=\left(R^{11}, R^{12}\right)$ & $R^{11}$ & $\rightarrow \quad R^{12}$ & & & & LP2 & LP3 \\
\hline$R^{2}=\left(R^{21}, R^{22}, R^{23}, R^{24}\right)$ & & $R^{24} \leftarrow$ & $-R^{23}$ & & & $-R^{22} \leftarrow$ & $R^{21}$ \\
\hline$R^{3}=\left(R^{31}, R^{32}\right)$ & & LP 1 & & & & $R^{32} \leftarrow$ & $R^{31}$ \\
\hline$R^{4}=\left(R^{41}, R^{42}\right)$ & & & & $R^{42}$ & & & $R^{41}$ \\
\hline$R^{5}=\left(R^{51}, R^{52}\right)$ & & & & & $R^{52}$ & & $-R^{51}$ \\
\hline
\end{tabular}

Figure 3: Five fragments recorded on seven traces.

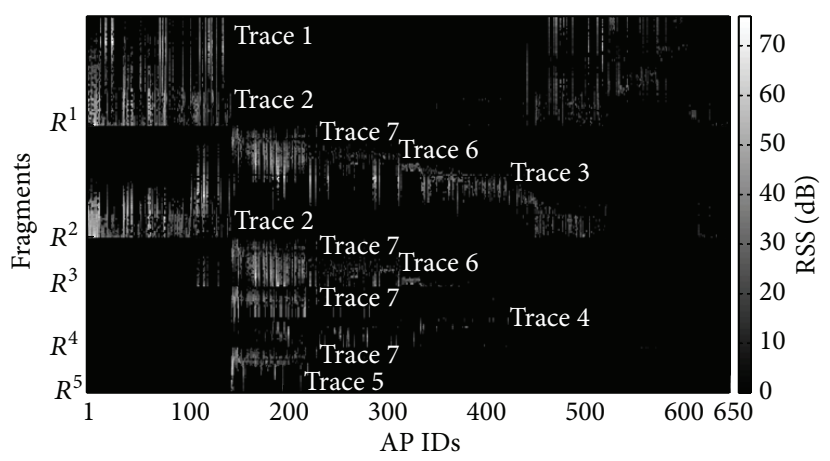

Figure 4: Raw measurement space.

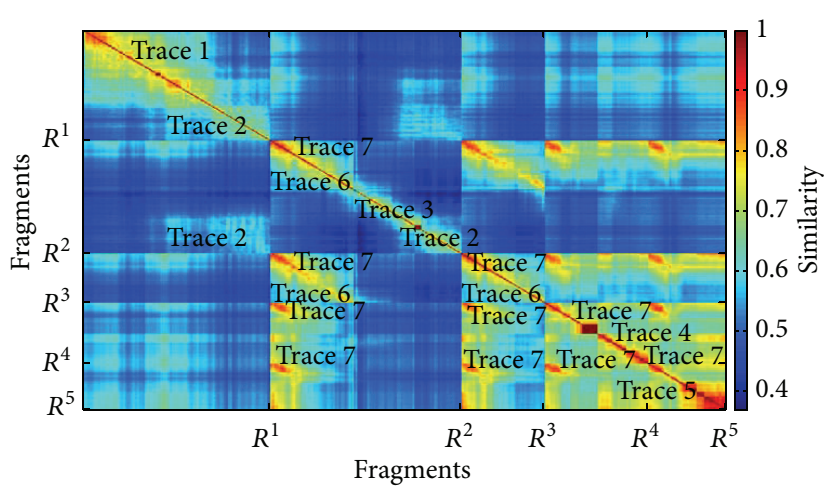

Figure 5: Similarity between any two measurements.

TABLE 4: Scores for the four most similar traces.

\begin{tabular}{|c|c|c|c|c|c|c|c|}
\hline \multirow{3}{*}{ Trace IDs } & \multicolumn{7}{|c|}{ New fragment IDs } \\
\hline & \multicolumn{2}{|c|}{ TP 1} & \multicolumn{2}{|c|}{ TP 2} & \multicolumn{3}{|c|}{ TP 3} \\
\hline & $\begin{array}{l}\text { Raw new } \\
\text { fragment }\end{array}$ & $\begin{array}{l}\text { 1st new } \\
\text { fragment }\end{array}$ & $\begin{array}{l}\text { Raw new } \\
\text { fragment }\end{array}$ & $\begin{array}{l}\text { 1st new } \\
\text { fragment }\end{array}$ & $\begin{array}{l}\text { Raw new } \\
\text { fragment }\end{array}$ & $\begin{array}{l}\text { 1st new } \\
\text { fragment }\end{array}$ & $\begin{array}{l}\text { 2nd new } \\
\text { fragment }\end{array}$ \\
\hline Trace 1 & 61 & 22 & 19.33 & 19.33 & l & l & l \\
\hline Trace 2 & 45.33 & 45.33 & 66.67 & 28 & 12 & 12 & I \\
\hline Trace 3 & I & I & I & I & 13.67 & 13.67 & 13.67 \\
\hline Trace 4 & l & l & l & l & l & l & 61.67 \\
\hline Trace 5 & 7 & l & l & l & 24 & 21 & 21 \\
\hline Trace 6 & 13.33 & 13.33 & 38 & 38 & l & l & l \\
\hline Trace 7 & l & 6.67 & 18.67 & 18.67 & 22 & 22 & 8.33 \\
\hline
\end{tabular}




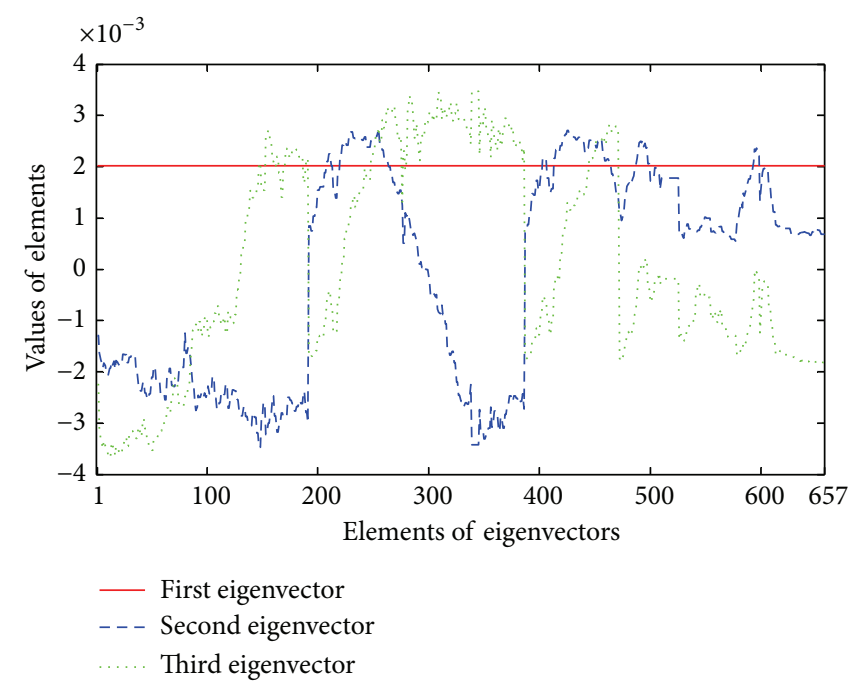

FIGURE 6: Eigenvectors for the construction of mapped low-dimensional space.

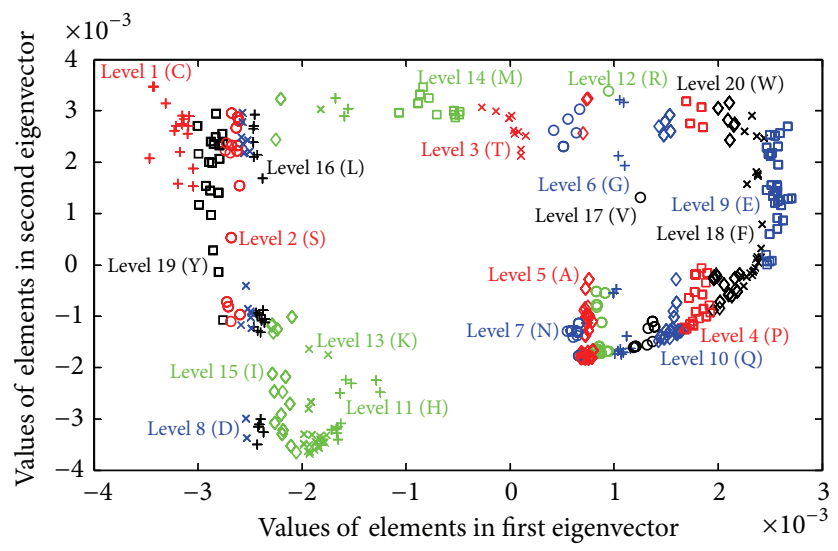

FIGURE 7: Measurement quantization into 20 different levels.

TABLe 5: Path tracking in mobility map.

\begin{tabular}{|c|c|}
\hline Actual motion paths & Tracking paths and new online fragments \\
\hline \multirow{2}{*}{$\begin{array}{l}\text { TP } 1 \\
(\text { Trace } 1 \rightarrow \text { Trace } 2)\end{array}$} & Tracking path: Trace $1 \rightarrow$ Trace 2 \\
\hline & $\begin{array}{l}\text { (i) Raw new fragment with the length of } 191 \\
\text { (measurement IDs from } 1 \text { to } 191 \text { ) } \\
\text { (ii) The 1st newly formed fragment with the length of } 87 \\
\text { (measurement IDs from } 105 \text { to 191) }\end{array}$ \\
\hline \multirow{2}{*}{$\begin{array}{l}\text { TP } 2 \\
(\text { Trace } 2 \rightarrow \text { Trace } 3 \rightarrow \text { Trace } 6 \rightarrow \text { Trace } 7 \text { ) }\end{array}$} & Tracking path: Trace $2 \rightarrow$ Trace 6 \\
\hline & $\begin{array}{l}\text { (i) Raw new fragment with the length of } 195 \\
\text { (measurement IDs from } 1 \text { to 195) } \\
\text { (ii) The 1st newly formed fragment with the length of } 142 \\
\text { (measurement IDs from } 54 \text { to 195) }\end{array}$ \\
\hline \multirow{2}{*}{$\begin{array}{l}\text { TP } 3 \\
(\text { Trace } 5 \rightarrow \text { Trace } 7 \rightarrow \text { Trace } 7 \rightarrow \text { Trace } 4 \text { ) }\end{array}$} & Tracking path: Trace $5 \rightarrow$ Trace $7 \rightarrow$ Trace 4 \\
\hline & $\begin{array}{l}\text { (i) Raw new fragment with the length of } 186 \\
\text { (measurement IDs from } 1 \text { to } 186 \text { ) } \\
\text { (ii) The 1st newly formed fragment with the length of } 148 \\
\text { (measurement IDs from } 39 \text { to } 186 \text { ) } \\
\text { (iii) The } 2 \text { nd newly formed fragment with the length of } 77 \\
\text { (measurement IDs from } 110 \text { to } 186 \text { ) }\end{array}$ \\
\hline
\end{tabular}




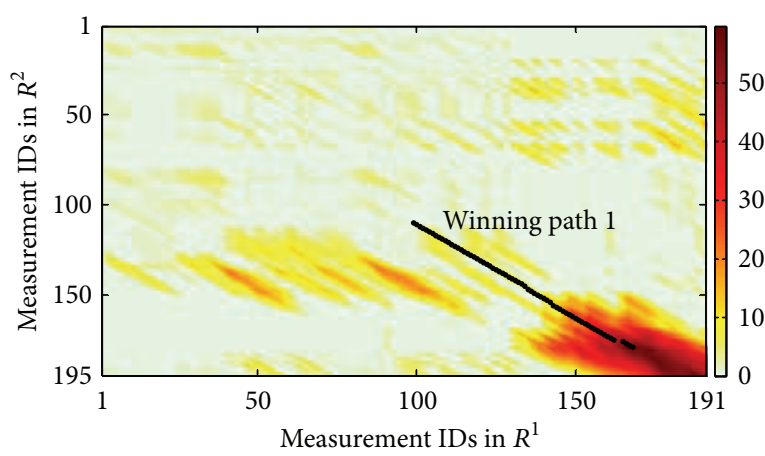

(a) Fragment pair $\left\{R^{1}, R^{2}\right\}$

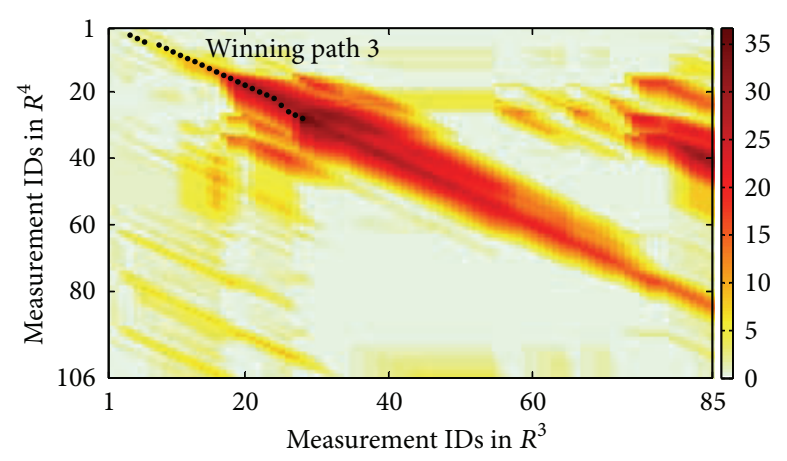

(c) Fragment pair $\left\{R^{3}, R^{4}\right\}$

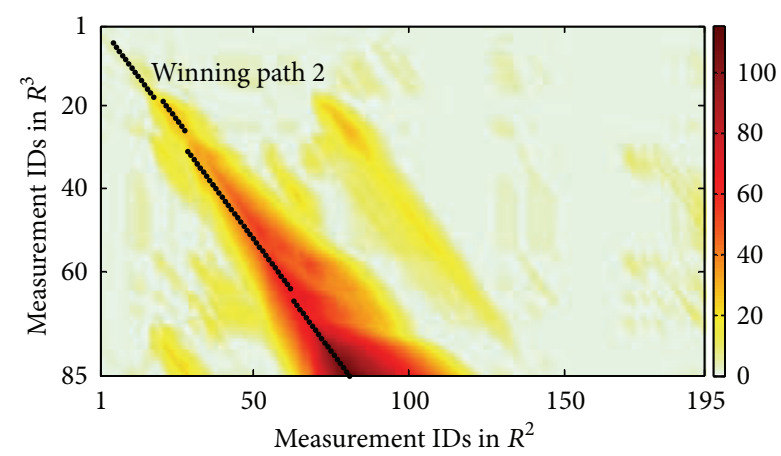

(b) Fragment pair $\left\{R^{2}, R^{3}\right\}$

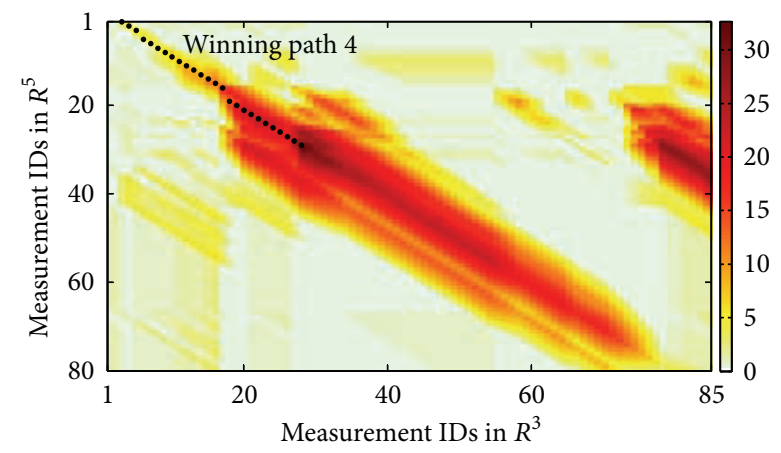

(d) Fragment pair $\left\{R^{3}, R^{5}\right\}$

FIGURE 8: Winning path in scoring space for each growing fragment pair.

Step (1): (i) winning path 1 generates LP1; (ii) $\operatorname{LP} 1\{\mathrm{~d}\} R^{1}$; and (iii) $\operatorname{LP} 1\{\mathrm{~d}\} R^{2}$ :

\begin{tabular}{ll|l|ll}
$R^{1}$ & Atrium & Library (191) & Lab (1) \\
\hline North Bus Stop (1) & Library (195) & LP1 & & $R^{2}$
\end{tabular}

Step (2): (i) winning path 2 generates LP2 and LP3; (ii) (LP2 and LP3) $\{\mathrm{s}\} R^{2}$; (iii) (LP2 and LP3) $\{=\} R^{3}$;

(iv) (LP2 and LP3) \{<\}LP1 in $R^{2}$; and (v) (LP2 and LP3) and LP 1 have the shared growing fragment $R^{2}$ :

\begin{tabular}{|c|c|c|c|c|c|}
\hline$R^{1}$ & Atrium & & & & \\
\hline North Bus Stop (1) & ibr & LP 1 & Library (191) & & $\mathrm{Lab}(1)$ \\
\hline & $y(110)$ & & Coffee Shop $(85)$ & LP2 and LP3 & \\
\hline
\end{tabular}

Step (3): (i) winning path 3 generates LP 3; (ii) $\operatorname{LP} 3\{\mathrm{~s}\} R^{3}$; (iii) $\operatorname{LP} 3\{\mathrm{~s}\} R^{4}$; and (iv) LP $3\{\mathrm{~s}\} \operatorname{LP} 2$ and LP3:

\begin{tabular}{|c|c|c|c|c|c|}
\hline$R^{1}$ & Atrium & & $\rightarrow$ Library (191) & & \\
\hline \multirow[t]{3}{*}{ North Bus Stop (1) } & J ihrary (195) & LP 1 & & Elevator & $\operatorname{Lab}(1)$ \\
\hline & & \multicolumn{3}{|c|}{ Coffee Shop $(85) \leftarrow$} & LP3 \\
\hline & & & Office $(106) \longleftarrow$ & & LP3 \\
\hline
\end{tabular}

Step (4): (i) winning path 4 generates LP5; (ii) $\operatorname{LP} 5\{\mathrm{~s}\} R^{3}$; (iii) $\operatorname{LP} 5\{\mathrm{~s}\} R^{5}$; and (iv) $\operatorname{LP} 5\{=\} \operatorname{LP} 3$ :

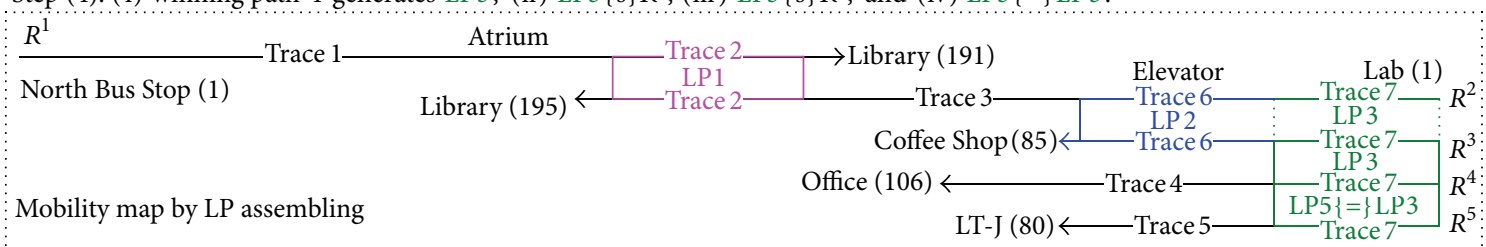

FIGURE 9: Mobility map constructed by LP assembling. 


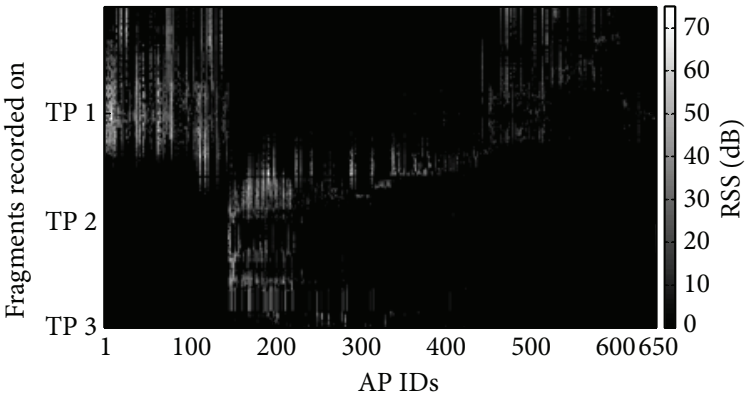

(a) New measurement space

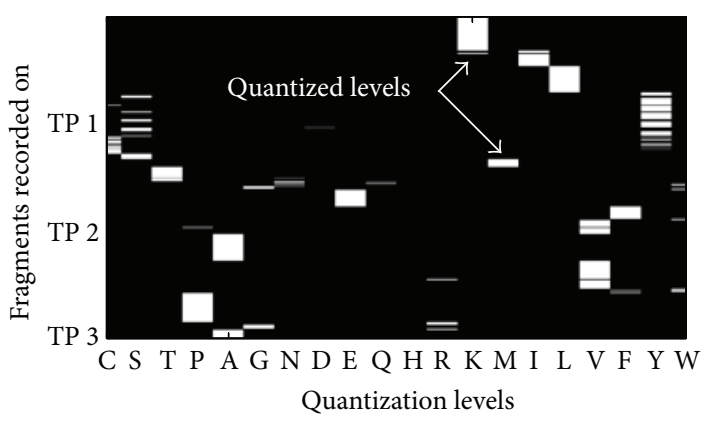

(b) Quantization results

FIGURE 10: Quantization for the new online fragments.
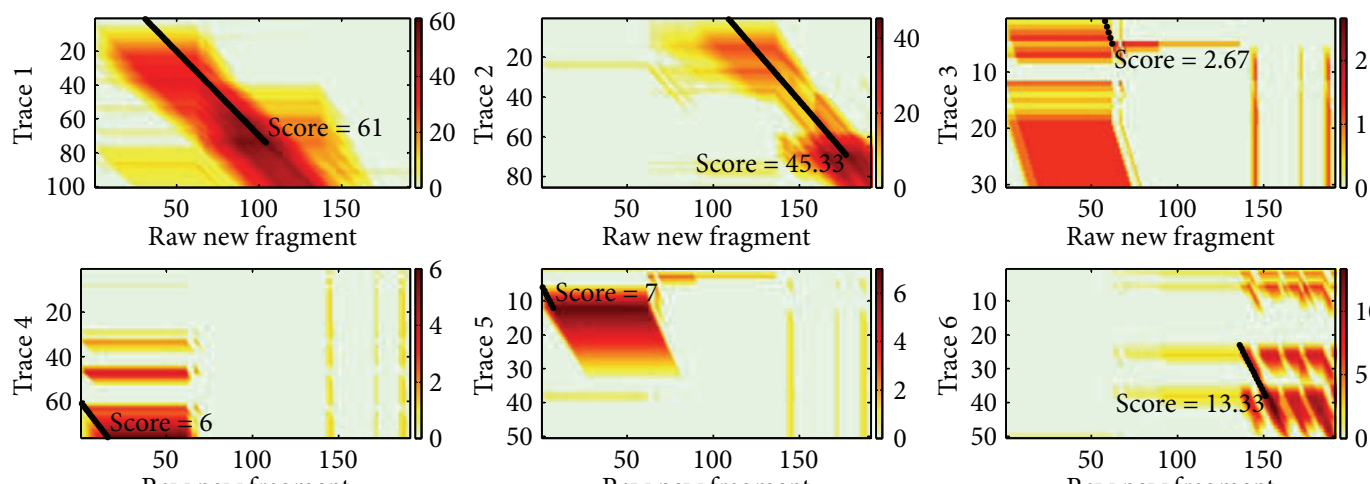

Raw new fragment

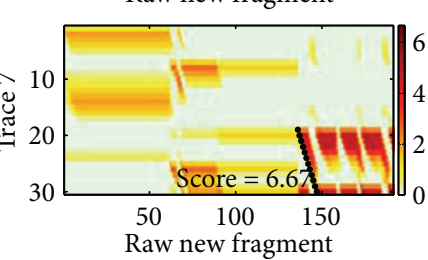

(a) Scoring spaces for the raw new fragment and labeling fragments on different traces
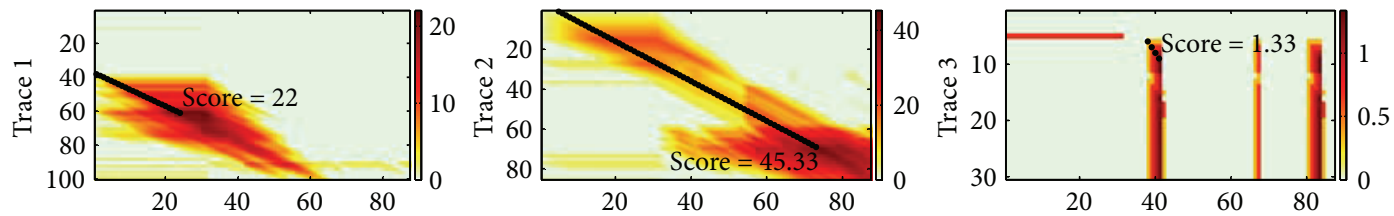

The 1st newly formed fragment

The 1st newly formed fragment

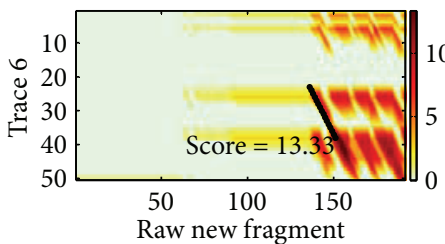

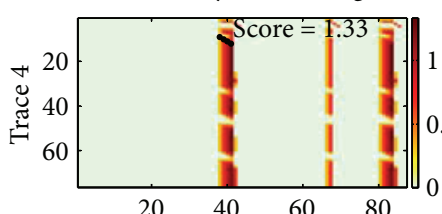

The 1st newly formed fragment

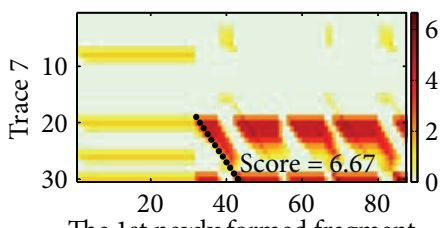

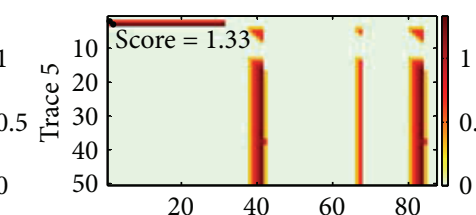

The 1st newly formed fragment

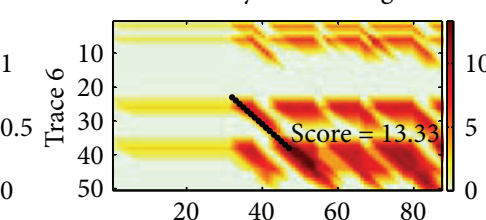

The 1st newly formed fragment

(b) Scoring spaces for the 1st newly formed fragment and labeling fragments on different traces

FIGURE 11: LP matching for the new online fragment recorded on TP 1. 


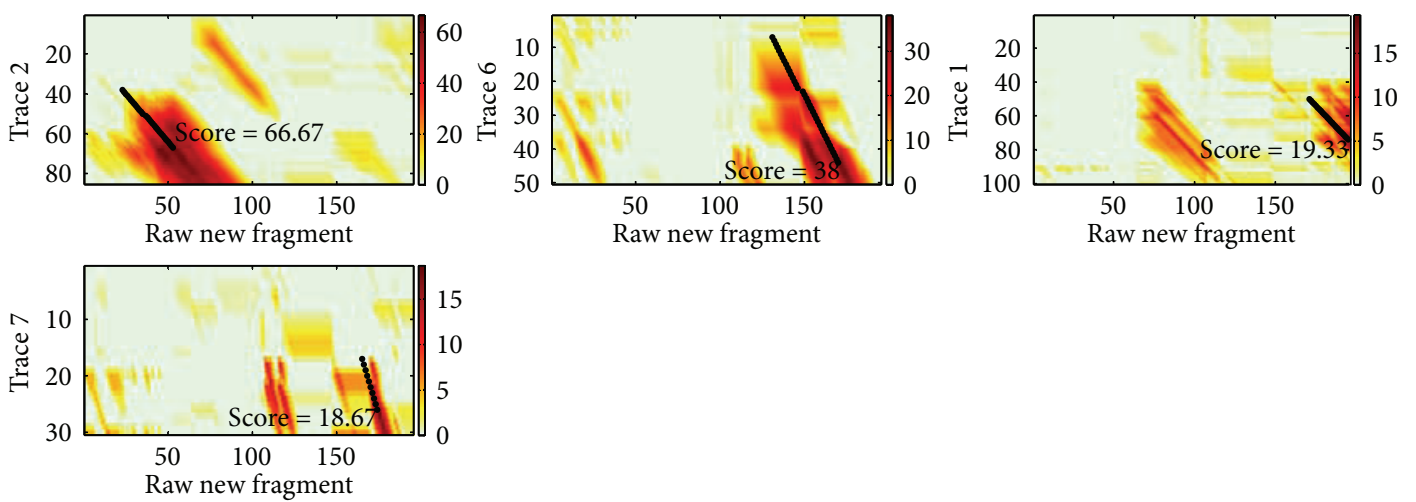

(a) Scoring spaces for the raw new fragment and labeling fragments on the four similar traces
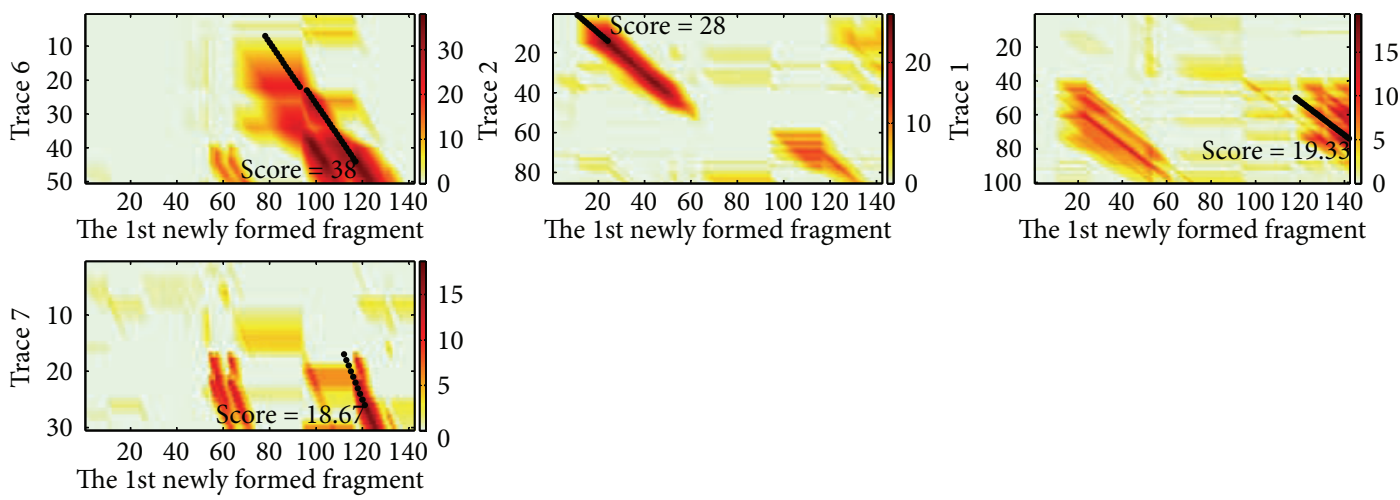

(b) Scoring spaces for the 1st newly formed fragment and labeling fragments on the four similar traces

FIGURE 12: LP matching for the new online fragment recorded on TP 2.

Met (M), Ile (I), Leu (L), Val (V), Phe (F), Tyr (Y), and Trp (W) [42]. After that, each RSS fragment can be represented by a string of amino acids, as shown in Table 3 .

Second, after the raw measurements are quantized into 20 discrete levels, we will calculate the winning paths from the scoring space for each growing fragment pair. By setting $\eta_{M}=$ 30 , we can obtain four winning paths from the four growing fragment pairs $\left\{R^{1}, R^{2}\right\},\left\{R^{2}, R^{3}\right\},\left\{R^{3}, R^{4}\right\}$, and $\left\{R^{3}, R^{5}\right\}$ in Figure 8 . The matching score, missing score, and mismatching score are determined by PAM250 mutation matrix [42]. In Figure 9, the mobility map associated with the target area is constructed by LP assembling. From Figures 3 and 9, the mobility map constructed by LP assembling is extremely similar to the trajectory of the people's actual motion. Then, the LP assembling is proved to perform well in layout description for the anonymous areas where the floor plans are not available.

Finally, to verify the efficiency of the mobility map constructed by LP assembling further, we use three paths for the testing of path tracking: (i) TP $1=$ Trace $1 \rightarrow$ Trace 2 which is the same as path 1 , (ii) TP $2=$ Trace $2 \rightarrow$ Trace $3 \rightarrow$ Trace $6 \rightarrow$ Trace 7 which is in the opposite direction of path 2, and (iii) TP $3=$ Trace $5 \rightarrow$ Trace $7 \rightarrow$ Trace $7 \rightarrow$ Trace 4 which is a combined path from paths 4 and 5 . The previous two traces on
TP 3 are in the opposite direction of path 5, while the following two traces are the same as path 4 . In coarse RSS quantization step, we quantize the new fragments recorded on TP 1 , TP 2 , and TP 3 (with the lengths of $N^{\mathrm{New}}=191,195$, and 186) into $\Phi=20$ discrete levels, as shown in Figure 10.

Then, after the new fragment recorded on each path has been quantized, we conduct the fine LP matching to identify the matching LPs in mobility map. Without loss of generality, all the traces in mobility map (i.e., Traces 1-7) are considered for LP matching in our experiments. Then, the traces with the corresponding labeling fragments which satisfy the relations in (8) are selected as the matching LPs. The results of LP matching for the new online fragments recorded on TP 1 , TP 2, and TP 3 are shown in Figures 11, 12, and 13, respectively.

In Figure 11(a), we find that the highest score, 61, is captured at the position $(74,104)$ in scoring space for the raw new fragment and the labeling fragment on Trace 1. Thus, we delete all the new measurements with the IDs not larger than $\tau^{*}=104$ from the raw new fragment to obtain the 1st newly formed fragment (with the measurement IDs from 105 to 191). As can be seen from Figure 11(b), Trace 2 with the highest score, 45.33 , to the 1st newly formed fragment is selected as the second matching LP. Since the scores for any traces to the 2nd newly formed fragment are lower than $\varepsilon_{S}$, we construct 

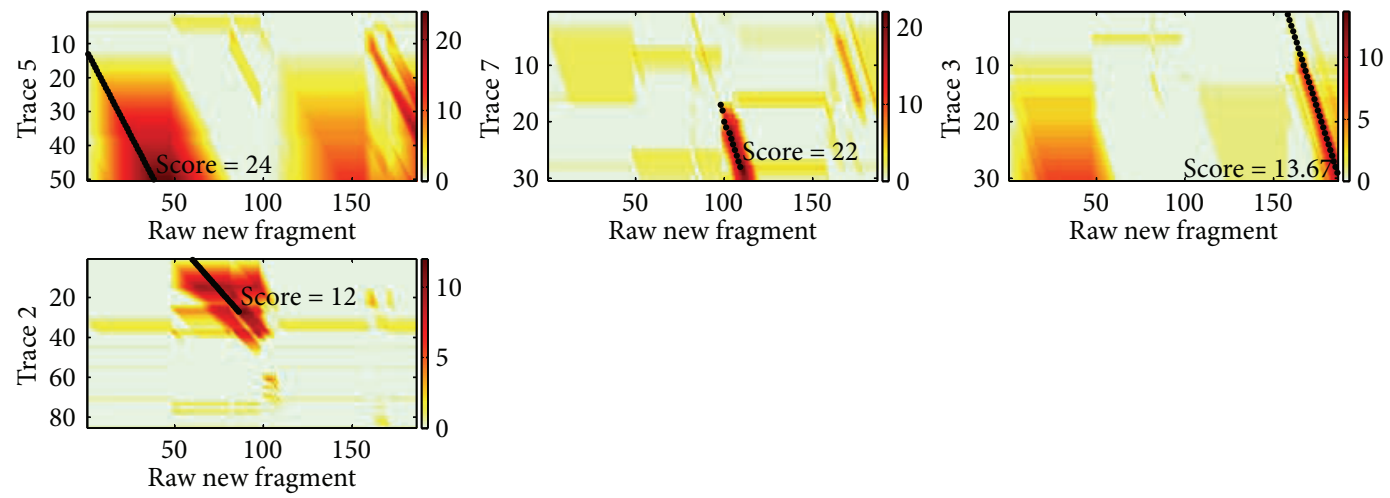

(a) Scoring spaces for the raw new fragment and labeling fragments on the four similar traces
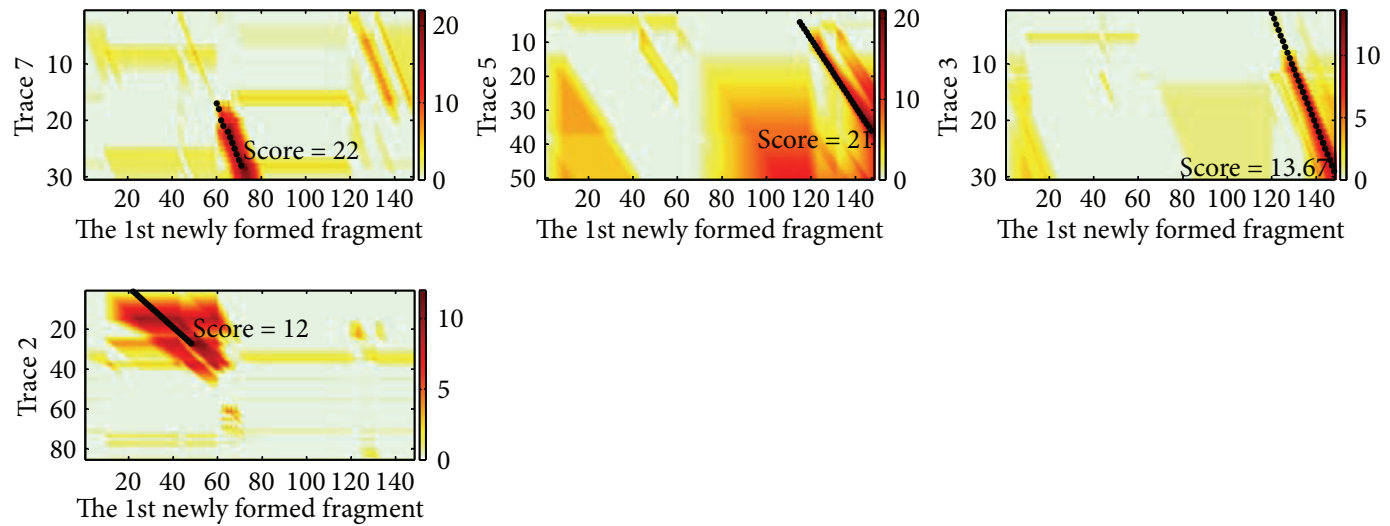

(b) Scoring spaces for the 1st newly formed fragment and labeling fragments on the four similar traces

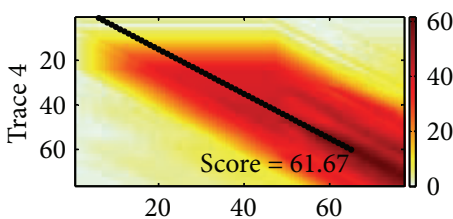

The 2nd newly formed fragment

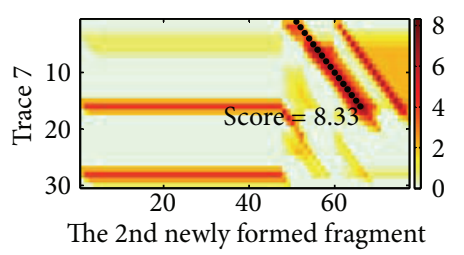

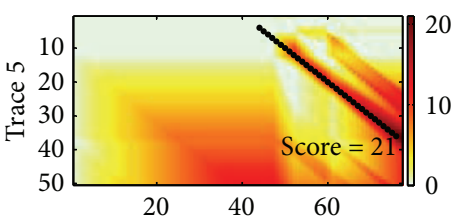

The 2nd newly formed fragment

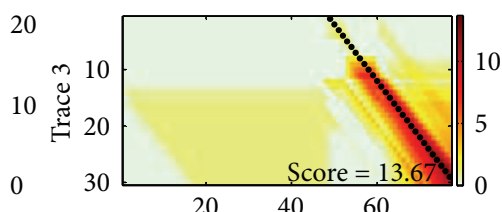

The 2nd newly formed fragment

(c) Scoring spaces for the 2nd newly formed fragment and labeling fragments on the four similar traces

FIGURE 13: LP matching for the new online fragment recorded on TP 3.

the people's motion path in mobility map as Trace $1 \rightarrow$ Trace 2 which is the same as the actual motion path, TP 1.

Figures 12 and 13 show the scoring spaces for the new online fragments recorded on TP 2 and TP 3 , respectively, and labeling fragments on the four most similar traces. The four traces which have the highest scores to the new online fragments are defined as the four most similar traces. The scores for the four most similar traces with respect to the new fragments are shown in Table 4.

Table 5 gives the new online fragments and the corresponding tracking paths which are connected by the matching LPs in mobility map. Therefore, our constructed timestamped mobility map performs well in people's motion path tracking, especially for the motion paths which are in the same direction of the raw paths used for mobility map construction. For instance, on TPs 1 and 3 , Trace $1 \rightarrow$ Trace 2 and Trace $7 \rightarrow$ Trace 4 which are in the same direction of the raw paths 1 and 4 are precisely tracked in mobility map in Figures 11 and 13.

\section{Conclusions}

In this paper, we introduced a novel Wi-Fi RSS measurement matching approach to construct a fine-granularity mobility map for layout description in anonymous areas without location fingerprinting. In our system framework, we first adopt 
Smith-Waterman alignment approach to obtain the significant LPs from measurement matching in scoring space. Then, Allen's interval algebra is used to conduct LP assembling into a time-stamped mobility map in temporal logic manner. Our extensive mathematical analysis and experimental results show that our approach can deal with the problems of mobility map construction from sporadically recorded highdimensional Wi-Fi RSS measurements and people's motion path tracking in arbitrary directions. We will continue to investigate the integration of time-stamped RSS measurements and motion sensors for the sake of constructing a highly precise mobility map. For instance, the relations of LPs can be described by not only the timestamps, but also the angle and speed measured by the off-the-shelf smartphones.

\section{Notation}

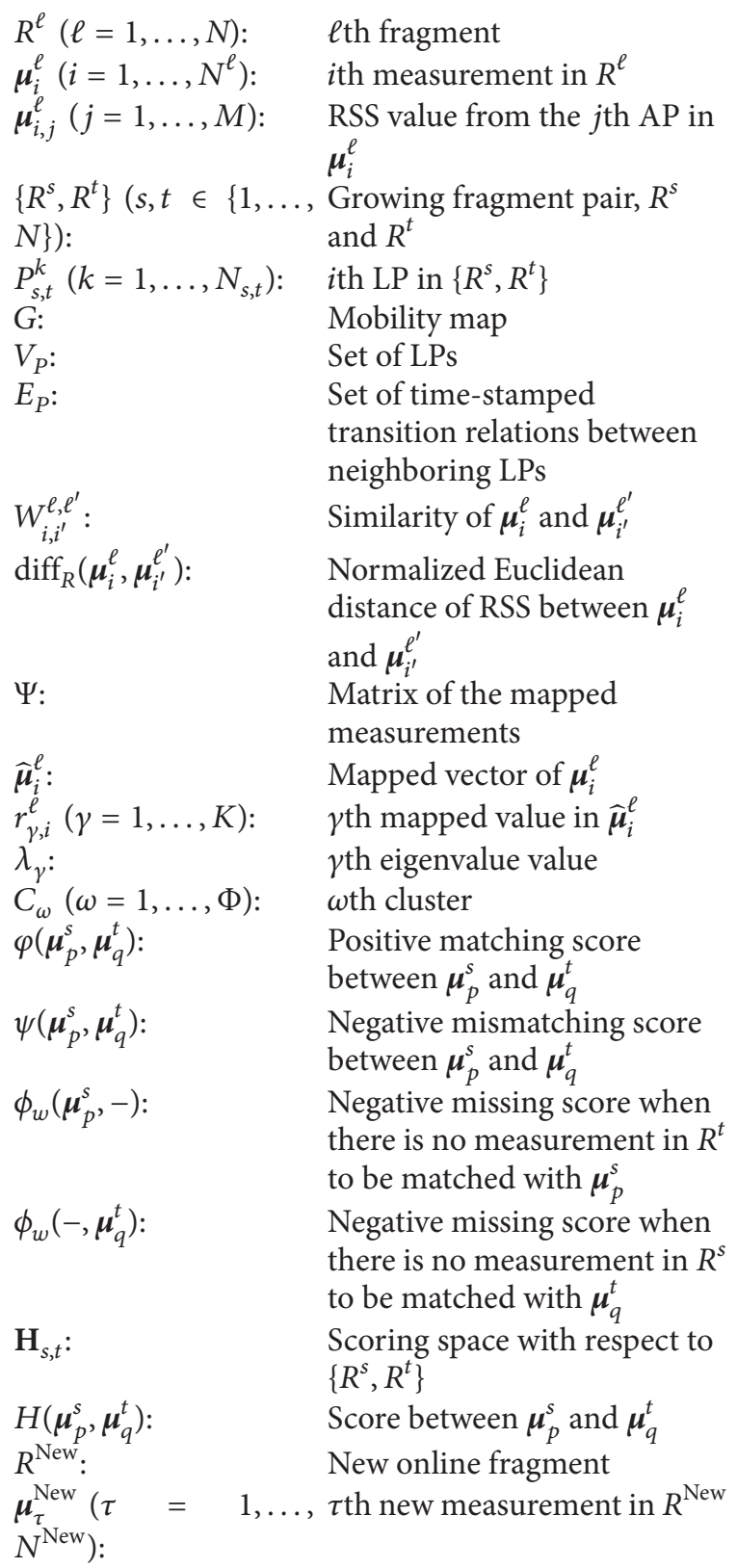

\begin{tabular}{|c|c|}
\hline $\begin{array}{l}\operatorname{Avg}\left(C_{\omega}\right)(\omega=1, \ldots, \\
\Phi):\end{array}$ & relage \\
\hline $\operatorname{diff}_{R}\left(\boldsymbol{\mu}_{\tau}^{\mathrm{New}}, \operatorname{Avg}\left(C_{k}\right)\right):$ & $\begin{array}{l}\text { Euclidean distance between } \\
\boldsymbol{\mu}_{\tau}^{\text {New }} \text { and } \operatorname{Avg}\left(C_{\omega}\right)\end{array}$ \\
\hline$R_{\omega}^{\ell}:$ & Labeling fragment in $C_{\omega}$ \\
\hline$N:$ & Number of fragments \\
\hline$N^{\ell}:$ & $\begin{array}{l}\text { Number of measurements in } \\
R^{\ell}\end{array}$ \\
\hline$M:$ & Number of hearable APs \\
\hline$N_{s, t}:$ & $\begin{array}{l}\text { Number of LPs with respect } \\
\text { to }\left\{R^{s}, R^{t}\right\}\end{array}$ \\
\hline$K:$ & $\begin{array}{l}\text { Number of dimensions of the } \\
\text { mapping space }\end{array}$ \\
\hline$\Phi:$ & $\begin{array}{l}\text { Number of clusters/number } \\
\text { of quantization levels }\end{array}$ \\
\hline$N_{\mathrm{LP}}:$ & Number of LPs after \\
\hline & $\begin{array}{l}\text { Smith-Waterman } \\
\text { measurement matching }\end{array}$ \\
\hline$N^{\text {New }}:$ & $\begin{array}{l}\text { Number of new } \\
\text { measurements in } R^{\mathrm{New}}\end{array}$ \\
\hline$N_{\omega}^{\ell}:$ & $\begin{array}{l}\text { Number of measurements } \\
\text { contained in } R_{\omega}^{\ell}\end{array}$ \\
\hline$\eta_{M}:$ & $\begin{array}{l}\text { Threshold for measurement } \\
\text { matching }\end{array}$ \\
\hline$\varepsilon_{S}:$ & $\begin{array}{l}\text { Threshold for the score of the } \\
\text { newly formed fragment }\end{array}$ \\
\hline$R_{P}=\left(\mathrm{LP}_{g}, \mathrm{LP}_{h}\right)$ & Temporal logic relation \\
\hline$\left.h=1, \ldots, N_{\mathrm{LP}}\right):$ & between the LPs $\mathrm{LP}_{g}$ and $\mathrm{LP}_{h}$ \\
\hline$\{=\},\{\mathrm{m}\},\{\mathrm{mi}\},\{\mathrm{o}\},\{\mathrm{oi}\}$, & 13 temporal logic relations in \\
\hline & \\
\hline ript “T”: & $\operatorname{Tr}$ \\
\hline & ce operation. \\
\hline
\end{tabular}

\section{Conflict of Interests}

The authors declare that there is no conflict of interests regarding the publication of this paper.

\section{Acknowledgments}

The authors wish to thank the reviewers and editors for their valuable comments and useful corrections to improve this work. This work was supported in part by the Program for Changjiang Scholars and Innovative Research Team in University (IRT1299), the National Natural Science Foundation of China (61301126 and 61304197), the National Science and Technology Major Project (2012ZX03006-002(3)), the Special Fund of Chongqing Key Laboratory (CSTC), the Fundamental and Frontier Research Project of Chongqing (cstc2013jcyjA40041, cstc2013jcyjA40032, and cstc2013jcyjA40034), the Scientific and Technological Research Program of Chongqing Municipal Education Commission (KJ130528), the Startup Foundation for Doctors of CQUPT (A2012-33), and the Science Foundation for Young Scientists of CQUPT (A2012-77). 


\section{References}

[1] Z. Chen, J. Xia, and B. Irawan, "Development of fuzzy logic forecast models for location-based parking finding services," Mathematical Problems in Engineering, vol. 2013, Article ID 473471, 6 pages, 2013.

[2] Z. Tian, X. Liu, M. Zhou, and K. Xu, "Mobility tracking by fingerprint-based KNN/PF approach in cellular networks," in Proceedings of the IEEE Wireless Communications and Networking Conference (WCNC '13), pp. 4570-4575, Shanghai, China, April 2013.

[3] J. Hightower and G. Borriello, "Location systems for ubiquitous computing," IEEE Computer, vol. 34, no. 8, pp. 57-66, 2001.

[4] H. Wu, T. Wang, and Z. Zhong, "Location updating schemes for high-speed railway cellular communication systems," Mathematical Problems in Engineering, vol. 2012, Article ID 802152, 15 pages, 2012.

[5] L. Zhang, F. Zhao, Z. Li, H. Luo, and J. Xu, "Design and implementation of WiFi network based mobile location service system," in Proceedings of the 2nd International Conference on Business Computing and Global Informatization (BCGIN '12), pp. 641-644, Shanghai, China, October 2012.

[6] C. Evans, "Intelligent retail business: location based services for mobile customers," in Proceedings of the 2nd International Conference on Pervasive Computing and Applications (ICPCA '07), pp. 354-359, Birmingham, UK, July 2007.

[7] T. R. Hansen, J. E. Bardram, and M. Soegaard, "Moving out of the lab: deploying pervasive technologies in a hospital," IEEE Pervasive Computing, vol. 5, no. 3, pp. 24-31, 2006.

[8] F. van Diggelen, "Indoor GPS theory \& implementation," in Proceedings of the IEEE Position Location and Navigation Symposium, pp. 240-247, Palms Springs, Calif, USA, April 2002.

[9] M. Zhou, Q. Guo, and Z. Wang, "A novel stable clustering design method for hierarchical satellite network," Chinese Journal of Aeronautics, vol. 23, no. 1, pp. 91-102, 2010.

[10] M. Zhou, Z. Tian, K. Xu, X. Yu, X. Hong, and H. Wu, "SCaNME: location tracking system in large-scale campus Wi-Fi environment using unlabeled mobility map," Expert Systems with Applications, vol. 41, no. 7, pp. 3429-3443, 2014.

[11] M. Zhao, H. Yang, J. Liu, Y. Chen, and J. Zhou, "Directional WiFi based indoor location system for emergency," in Proceedings of the 7th International Conference on Ubiquitous Intelligence \& Computing/Autonomic \& Trusted Computing, pp. 501-502, Xian, China, October 2010.

[12] M. Zhou, Z. Tian, K. Xu, X. Yu, and H. Wu, “Theoretical entropy assessment of fingerprint-based Wi-Fi localization accuracy," Expert Systems with Applications, vol. 40, no. 15, pp. 6136-6149, 2013.

[13] M. Zhou, Z. Tian, K. Xu, X. Hong, and X. Tang, “Towards statistical linear accuracy of neighbor matching localization in Wi-Fi environment," Journal of Computational Information Systems, vol. 9, no. 22, pp. 9177-9184, 2013.

[14] K. Kaemarungsi and P. Krishnamurthy, "Properties of indoor received signal strength for WLAN location fingerprinting," in Proceedings of the 1st Annual International Conference on Mobile and Ubiquitous Systems: Networking and Services (MOBIQUITOUS '04), pp. 14-23, Boston, Mass, USA, August 2004.

[15] S.-H. Fang and T.-N. Lin, "A dynamic system approach for radio location fingerprinting in wireless local area networks," IEEE Transactions on Communications, vol. 58, no. 4, pp. 1020-1025, 2010.
[16] M. Zhou, Y. Xu, L. Ma, and S. Tian, "On the statistical errors of RADAR location sensor networks with built-in Wi-Fi Gaussian linear fingerprints," Sensors, vol. 12, no. 3, pp. 3605-3626, 2012.

[17] Z. Deng, Y. Xu, and L. Ma, "Indoor positioning via nonlinear discriminative feature extraction in wireless local area network," Computer Communications, vol. 35, no. 6, pp. 738-747, 2012.

[18] M. Zhou, Y. Xu, and L. Tang, "Multilayer ANN indoor location system with area division in WLAN environment," Journal of Systems Engineering and Electronics, vol. 21, no. 5, pp. 914-926, 2010.

[19] C. Koweerawong, K. Wipusitwarakun, and K. Kaemarungsi, "Indoor localization improvement via adaptive RSS fingerprinting database," in Proceedings of the International Conference on Information Networking (ICOIN '13), pp. 412-416, Bangkok, Thailand, January 2013.

[20] X. Wang, A. K. Wong, and Y. Kong, "Mobility tracking using GPS, Wi-Fi and cell ID," in Proceedings of the the International Conference on Information Networking (ICOIN '12), pp. 171-176, Phuket, Thailand, February 2012.

[21] M. Zhou, A. K. Wong, Z. Tian, V. Y. Zhang, X. Yu, and X. Luo, "Adaptive mobility mapping for people tracking using unlabelled Wi-Fi shotgun reads," IEEE Communications Letters, vol. 17, no. 1, pp. 87-90, 2013.

[22] T. F. Smith and M. S. Waterman, "Identification of common molecular subsequences," Journal of Molecular Biology, vol. 147, no. 1, pp. 195-197, 1981.

[23] J. F. Allen, "Maintaining knowledge about temporal intervals," Communications of the ACM, vol. 26, no. 11, pp. 832-843, 1983.

[24] M. Zhou, Y. Xu, and L. Ma, "Radio-map establishment based on fuzzy clustering for WLAN hybrid KNN/ANN indoor positioning," China Communications, vol. 7, no. 3, pp. 64-80, 2010.

[25] Z. Tian, X. Tang, M. Zhou, and Z. Tan, "Fingerprint indoor positioning algorithm based on affinity propagation clustering," EURASIP Journal on Wireless Communications and Networking, vol. 2013, article 272, 2013.

[26] E. Flannery, A. Haeberlen, L. Kavraki, A. Ladd, A. Rudys, and D. Wallach, "System and method for localization over a wireless network," Patent WO 2006017266 A2, 2006.

[27] M. Paciga and H. Lutfiyya, "Herecast: an open infrastructure for location-based services using WiFi," in Proceedings of the IEEE International Conference on Wireless and Mobile Computing, Networking and Communications (WiMob '05), vol. 4, pp. 21-28, Montreal, Canada, August 2005.

[28] P. Bahl and V. N. Padmanabhan, "RADAR: an in-building RFbased user location and tracking system," in Proceedings of the 19th IEEE Annual Joint Conference of the IEEE Computer and Communications Societies (IEEE INFOCOM '00), pp. 775-784, Tel-Aviv, Israel, March 2000.

[29] P. Castro, P. Chiu, T. Kremenek, and R. Muntz, "A probabilistic room location service for wireless networked environments," in Proceedings of the 3rd International Conference on Ubiquitous Computing (UbiComp '01), pp. 18-34, Atlanta, Ga, USA, March 2001.

[30] M. Youssef and A. Agrawala, "The Horus location determination system," Wireless Networks, vol. 14, no. 3, pp. 357-374, 2008.

[31] D. Kurt and M. Milos, "Wireless based object tracking based on neural networks," in Proceedings of the 3rd IEEE Conference on Industrial Electronics and Applications (ICIEA '08), pp. 308-313, Singapore, June 2008. 
[32] S.-H. Fang and T.-N. Lin, "Indoor location system based on discriminant-adaptive neural network in IEEE 802.11 environments," IEEE Transactions on Neural Networks, vol. 19, no. 11, pp. 1973-1978, 2008.

[33] R. Battiti, T. L. Nhat, and A. Villani, "Location-aware computing: a neural network model for determining location in wireless LANs," Tech. Rep. DIT-02-0083, Department of Information and Communication Technology, University of Trento, Trento, Italy, 2002.

[34] S. Saha, K. Chaudhuri, D. Sanghi, and P. Bhagwat, "Location determination of a mobile device using IEEE 802.11b access point signals," in Proceedings of the IEEE Wireless Communications and Networking (WCNC '03), vol. 3, pp. 1987-1992, New Orleans, La, USA, March 2003.

[35] S. A. Golden and S. S. Bateman, "Sensor measurements for WiFi location with emphasis on time-of-arrival ranging," IEEE Transactions on Mobile Computing, vol. 6, no. 10, pp. 1185-1198, 2007.

[36] A. V. Alejos, M. G. Sanchez, D. R. Iglesias, and I. Cuinas, "Incidence angle estimation algorithm for a low-cost AoA BASED INDOOR location system," in Proceedings of the IEEE International Symposium on Antennas and Propagation and USNC/ URSI National Radio Science Meeting (APSURSI '09), pp. 1-4, Charleston, SC, USA, June 2009.

[37] A. Narzullaev, Y. W. Park, and H. Y. Jung, "Accurate signal strength prediction based positioning for indoor WLAN systems," in Proceedings of the IEEE/ION Position, Location and Navigation Symposium (PLANS '28), pp. 685-688, Monterey, Calif, USA, May 2008.

[38] M. Zhou, Z. Tian, K. Xu, X. Yu, and H. Wu, "Error analysis for RADAR neighbor matching localization in linear logarithmic strength-varying Wi-Fi environment," The Scientific World Journal, vol. 2014, Article ID 647370, 15 pages, 2014.

[39] H. Alasti, K. Xu, and Z. Dang, "Efficient experimental path loss exponent measurement for uniformly attenuated indoor radio channels," in Proceedings of the 10th IEEE Southeast Conference (SOUTHEASTCON '09), pp. 255-260, Atlanta, Ga, USA, March 2009.

[40] M. Belkin and P. Niyogi, "Laplacian eigenmaps for dimensionality reduction and data representation," Neural Computation, vol. 15, no. 6, pp. 1373-1396, 2003.

[41] http://pathadvisor.ust.hk.

[42] M. O. Dayhoff, R. M. Schwartz, and B. C. Orcutt, "A model of evolutionary change in proteins," Atlas of Protein Sequence and Structure, vol. 5, supplement 3, pp. 345-351, 1978. 


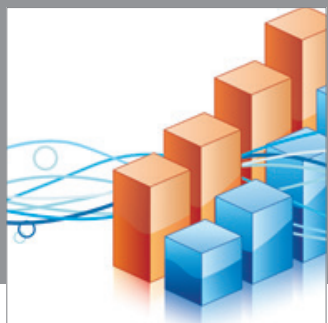

Advances in

Operations Research

mansans

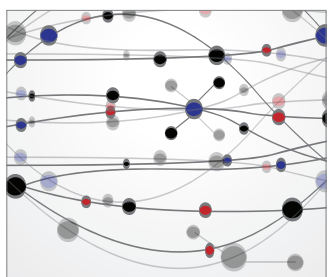

The Scientific World Journal
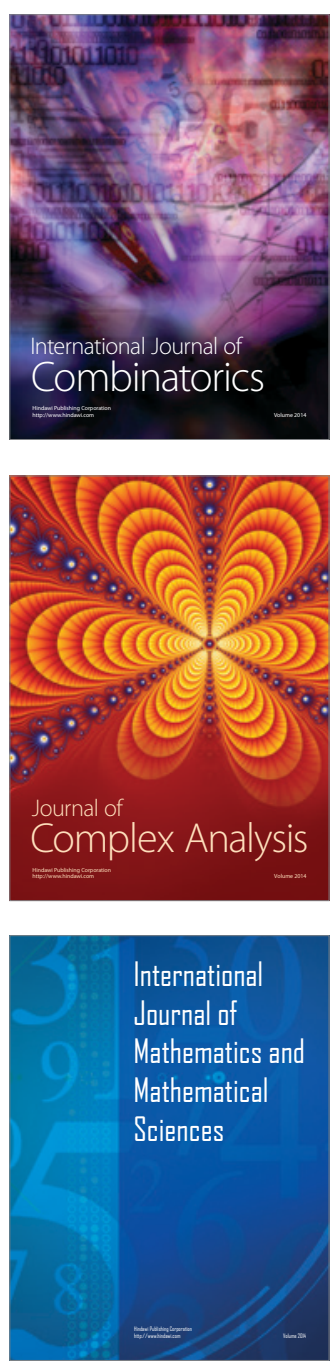
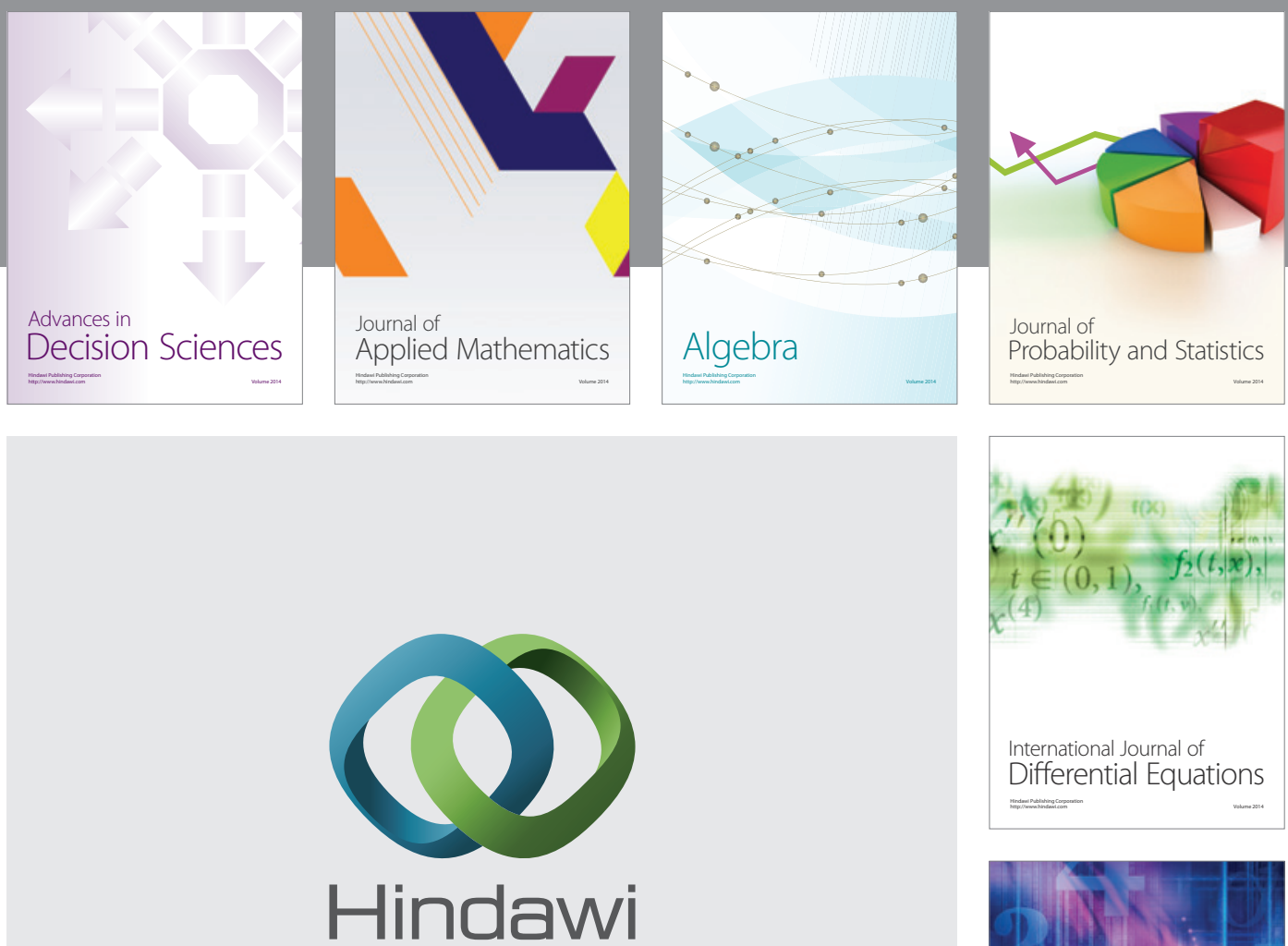

Submit your manuscripts at http://www.hindawi.com
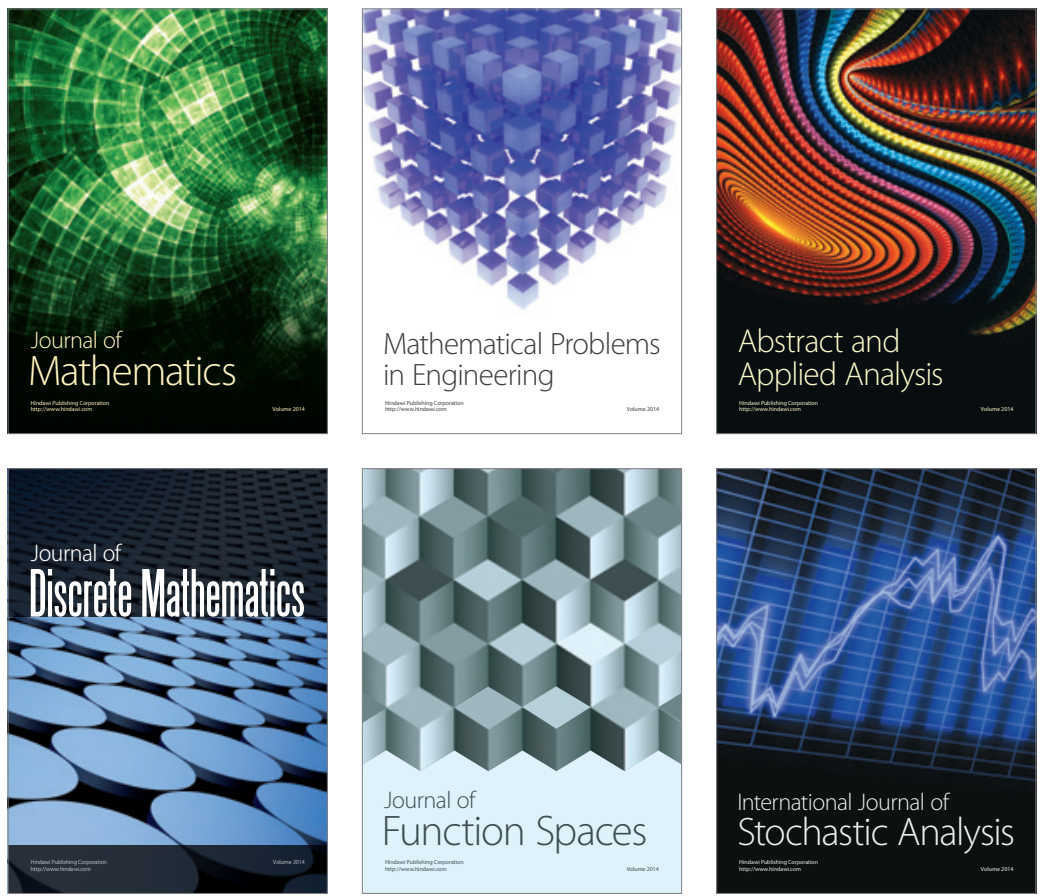

Journal of

Function Spaces

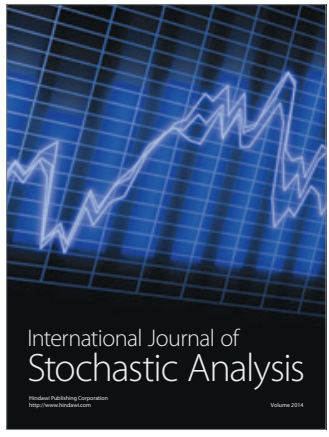

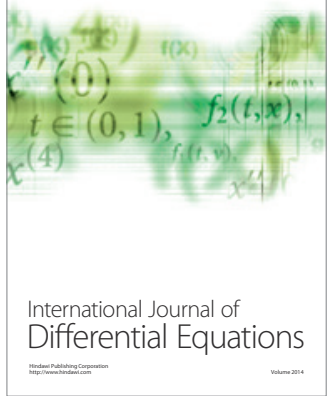
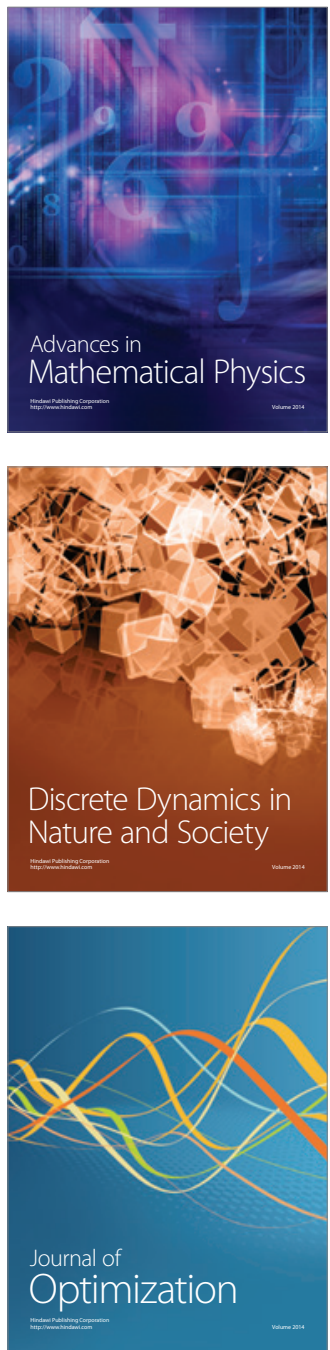